\title{
Theoretical study of soft X-ray emission and dynamics of laser-induced plasma of double stream gas puff
}

\author{
Vassily S Zakharov ${ }^{1,2,3, a}$ (D) and Sergey V Zakharov ${ }^{1,2}$ \\ 1 EATS, Orsay, France \\ 2 NRC Kurchatov Institute, Moscow, Russia \\ 3 Keldysh Institute of Applied Mathematics RAS, Moscow, Russia
}

Received 30 March 2021 / Accepted 21 October 2021 / Published online 12 November 2021 (C) The Author(s) 2021

\begin{abstract}
The double stream gas puff-based laser-produced plasma is studied as a source of soft X-ray radiation in $\mathrm{nm}$ wavelength spectral range. Dynamics of plasma induced by Nd:YAG laser beam and its emission is simulated with radiation-magnetohydrodynamic code Zstar. The modeling results for krypton gas stream in an annular helium jet as a circumferential gas for various picosecond and nanosecond laser pulses corresponding to the experiments are presented. The spatial-spectral features and temporal behavior of the soft X-ray and EUV emission are investigated. Under ps pulse, the gas is rapidly ionized in the laser beam channel, but it does not have enough time to shift sensibly during the pulse, and the plasma electron density grows against the background of almost constant ion density during the ionization in the laser radiation field. There is ionization instability only capable to be developed in ps range. At ns pulse, the gas ionization and heating leads to gas pushing out of the channel, and the formation of a divergent compression wave transforming into the shock wave. Behind the compression wave front, conditions arise for the development of Rayleigh-Taylor-type instability. The instability leads to the redistribution of plasma temperature and density, and to the formation of increased soft X-ray emission spots. Time evolution of spatial distributions and spectral characteristics of emitted SXR radiation is analyzed for different laser pulses. Transient effects in multicharged ion plasma are discussed, fundamental understanding of those is required for optimization of plasma radiation source. A conversion efficiency of laser energy into soft X-ray wavebands from krypton plasma is scanned by laser parameters and analyzed.
\end{abstract}

\section{Introduction}

The high brightness extreme-ultra-violet (EUV) and soft X-ray (SXR) plasma emission, particularly in the water-window spectral range from 2.33 to $4.4 \mathrm{~nm}$ wavelength, is of great interest in academic research on non-equilibrium radiative processes in the matter and in possible plasma applications for biomedical SXR microscopy [1-4], material processing [5], near edge $\mathrm{x}$ ray absorption spectroscopy [6-8], scintillation [9], EUV and SXR lithography [10,11], actinic inspection [12], metrology [13], etc. SXR and EUV in the wavelength ranging from about $1 \mathrm{~nm}$ to $50 \mathrm{~nm}$ can be efficiently emitted from the plasma generated by interaction of laser pulses with matter. Dense and hot laser-produced plasma (LPP) emitting SXR may be created either in a gas or on a surface of a solid- or liquid-state target [1417]. The target substance, heated by monochromatic low-energy photon but intensive laser light, passes to a plasma state through a sequence of quantum, collective and subsequent thermalization effects. The plasma reemits the absorbed laser energy in a wide range EUV/SXR spectrum depending on its composition.

\footnotetext{
${ }^{\mathrm{a}}$ e-mail: zakharovvas@gmail.com (corresponding author)
}

However, the conventional laser plasma sources based on a solid/liquid target have serious debris production problem. To avoid this problem gas puff targets produced by means of nozzles are applied for LPP SXR and EUV radiation sources. Recent experiments with gas puff targets showed that emission generated from laser plasma created by picosecond laser pulses is more efficient when compared to nanosecond laser pulses [1719]. A higher conversion efficiency achieved in interaction of shorter laser pulses with nitrogen gas puff targets has already been explained by detailed computer simulations of experiments with nitrogen gas puff and different pulse durations [20]. If the nitrogen gas is used, only a few distinct spectral lines from Be- to H-like ions are positioned in the water-window spectral band and below [21]. This limits the conversion efficiency into the emission in spectral band required.

The laser plasma generated in gas puff targets with higher atomic numbers (e.g., argon, krypton or xenon) emits the radiation consisting of many overlapping lines providing like unresolved transition array spectra. If the plasma is created by picosecond laser pulse, the peak value of the spectral intensity is considerably shifted towards shorter wavelengths compared to the position 
of the peak value achieved with nanosecond laser pulse excitation [22].

To improve the debris-free property of gas puff and to suppress in part the radial expansion of the target gas-jet, a double-stream gas puff target created by injection of high-Z driver gas (xenon, krypton, argon, etc.) into a hollow stream from low-Z gas as helium has been proposed [15]. Due to the shielding effect, the buffering helium stream efficiently suppresses the amount of debris. At the same time, due to low-Z, the buffering helium stream is almost transparent for SXR and EUV emission of the high-Z driver gas and doesn't reduce the conversion efficiency.

Strong SXR and EUV emissions from laser plasma based on a double-stream gas puff have been demonstrated [23] and have been used in variety of applications in science and technology. The source was successfully applied for construction of EUV and soft Xray microscopes with sub-100 $\mathrm{nm}$ resolution $[1,3]$. A compact source dedicated for application in metrology [13] has been developed and used for testing of optical elements for SXR and EUV ranges. The laser plasma EUV/ SXR source dedicated for material processing and photo-induced plasma studies has been also developed [6]. It was recently used for construction of new setups dedicated for soft X-ray absorption spectroscopy and the optical coherent tomography using soft X-rays.

SXR and EUV generations were widely and carefully studied experimentally, while there are few results only on theoretical research and computer simulations. Theoretical studies and computer simulations of the radiation generation process using plasma codes are strongly required.

The high-energy density effects based particularly on laser pulse action on a target required are used to produce the radiating plasma. Heated up by intensive laser light, the target gas passes to a plasma state. The plasma gets hot, moves fast and reemits a part of the absorbed laser energy in a wide range EUV/SXR spectrum depending on its composition. Such transient LPP itself is in non-equilibrium state.

The absolute value of conversion efficiency of the laser light into EUV/SXR or harder radiation range depends on a number of laser parameters in a complicated manner, e.g., under a given laser intensity the conversion efficiency increases with laser energy even for longer laser pulse or wider laser focusing. This is a result of transient effects in multicharged ion plasma which requires a fundamental understanding for optimization of a plasma radiation source.

In the presented paper, the theoretical aspects of the collaboration with experimental group at Institute of Optoelectronics, Military University of Technology, Warsaw, Poland, are considered on physics of emission properties and dynamics of LPP of double stream gas-puff in a wide range of Nd:YAG laser pulse energy and pulse duration. Accurate numerical modeling with the help of radiation-magnetohydrodynamics (RMHD) numerical code Zstar of transient non-equilibrium plasmas self-consistently with ionization phenomena and radiation transfer are performed, and conversion effi- ciencies in SXR spectral ranges at various laser parameters are compared. The time resolved detailed line emission spectrum in the water-window spectral range is estimated using post-processing based on calculations of atomic structures and level populations in ions upon the Hartree-Fock-Slater (HFS) quantumstatistical model accounting all possible transitions between the levels including single- and double-excited states.

\section{Modeling of double-stream gas puff with Zstar code}

The RMHD plasma code Zstar [24] is applied to simulate self-consistently the plasma dynamics with ionization effects and radiation transport in laser and discharge plasmas. Zstar is a 2-D computational code in cylindrical geometry designed to focus specifically in the simulation of a multicharged ion plasma in experimental and industrial facilities. It uses either a radiative magnetohydrodynamic approach to simulate dynamics of quasi-neutral plasma or electron-hydrodynamic (or particle-in-cell) approach to simulate flows of electrons in a weakly ionized gas. In RMHD approach, the mathematical model is based on a completely conservative, implicit difference scheme in Euler-Lagrange variables, when the main conservation laws (mass, energy, momentum, angular momentum) are fulfilled automatically in the system. Plasma radiation properties, ionization and equation of state (EOS), as well as excitation and ionization rates, and plasma kinetic coefficients are calculated by means of interpolations from a set of tables prepared in pre-processing with the Hartree-Fock-Slater (HFS) model [25] and ionization kinetics [24] in both the optically thick LTE, and the transparent non-LTE limits. The actual non-LTE condition at any instant is modeled by analytical interpolation between these two limits [26]. The radiation field in the quasi-stationary approximation is found by integrating the radiation transport equation along the trajectories under cylindrical symmetry conditions [27]. The non-equilibrium radiation physics with selfabsorption/emission is included in this code. Emission characteristics in selected spectral ranges may be followed.

The laser light transport is calculated by means of two-direction transfer model [28] taking into account an absorption and reflection of the laser light along its trajectories. The laser light absorption coefficient includes an interaction of radiation with electrons, ions and neutral atoms: by means of collisional (inversebremsstrahlung) absorption with plasma dispersion properties [29], resonant absorption (a critical density effect due to Langmuir plasma oscillation resonant excitation) [29]. The effective bound-bound excitation calculated from spectral tables at laser quantum energy with taking into account the induced deexcitation and a probability of dissipation of absorbed laser energy 
to thermal energy, direct ionization (if laser quantum energy is higher than the photo-ionization threshold) calculated from spectral tables or tunnel ionization (if laser quantum energy is lower than the ground state ionization energy) [30] are included in the model as well. More detailed description on models, numerical methods and difference schemes on solvers implemented in Zstar RMHD code, database contents and various examples of simulations as well can be found in $[24,31]$.

Actual simulations were done for LPP EUV and SXR radiation source with double stream gas-puff of $\mathrm{He}: \mathrm{Kr}$ and other gases under Nd:YAG laser (with light wavelength of $1.064 \mathrm{~nm}$ ) interaction with various laser pulse durations and pulse energies. The input parameters of laser and double gas-puff stream in Fig. 1 are chosen according to the experiments performed at the Institute of Optoelectronics at MUT in Warsaw [32]. The gas streams are approximated by gas layers. The thickness of the layers is chosen to be equal to the diameter of the gas streams at the position of interaction with the laser beam, i.e., the krypton layer is estimated as $1.2 \mathrm{~mm}$ thickness between two helium layers of $0.9 \mathrm{~mm}$ depth each. The initial gas pressure in the layers of krypton and helium was chosen as equal to each other and was defined by optimization of SXR emission. The initial mass density of krypton gas for picosecond laser pulses was of $2.9 \cdot 10^{-4} \mathrm{~g} / \mathrm{cm}^{3}$ and for nanosecond laser pulses was of $5.8 \cdot 10^{-4} \mathrm{~g} / \mathrm{cm}^{3}$. These initial gas densities were chosen as uniform in the layers. In the simulated geometry, the axis of symmetry coincides with the laser beam axis $\mathrm{Z}$.

The considered laser energies varied from $500 \mathrm{~mJ}$ to $8 \mathrm{~J}$ and the pulse durations with full width half maximum (FWHM) at Gaussian temporal profiles were taken from $150 \mathrm{ps}$ to $10 \mathrm{~ns}$. The laser beam has a Gaussian radial profile and propagates in the opposite direction to the $\mathrm{Z}$ axis, it is focused (with $25 \mathrm{~cm}$ focal length) into the center of krypton gas layer with focal spot diameter from $60 \mu \mathrm{m}$ to $400 \mu \mathrm{m}$ FWHM. The laser power considered provides a laser light intensity of the order of $\mathrm{TW} / \mathrm{cm}^{2}$ in the focal spot. Four sets of parameters such as laser energy, pulse duration and focusing degree simulated were considered below to demonstrate the key physical features at He:Kr double stream gaspuff LPP conditioning the plasma emission in different SXR spectral ranges.

\section{Simulation results and discussion}

\subsection{Picosecond laser plasma dynamics}

The incident laser beam of $\mathrm{TW} / \mathrm{cm}^{2}$ intensity causes the fast gas ionization due to the tunnel effect, an efficient acceleration of free electrons and a high rate of their collisions with atoms, which lead to a laser-plasma discharge development and fast plasma formation. The laser light absorption increases self-consistently with ionization. The laser energy is absorbed in the kryp- ton layer mainly. As it follows, the plasma temperature and its pressure increase.

3.1.1 Results on laser with energy $500 \mathrm{~mJ}$, pulse $150 \mathrm{ps}$, intensity $1.67 \mathrm{TW} / \mathrm{cm} 2$

At picosecond times, plasma ions do not have enough time to shift significantly, the plasma electron density grows against the background of almost constant ion density due to ionization in the laser radiation field. This effect can be seen in Fig. 2, which shows: (a) plasma mass-density and (b) electron density distributions at $300 \mathrm{ps}$, corresponding to the plasma SXR radiation peak for the laser pulse of energy $500 \mathrm{~mJ}$. The laser pulse duration is $150 \mathrm{ps}$ FWHM focused into the spot of $400 \mu \mathrm{m} \mathrm{FWHM}$ in the center of the krypton layer, which corresponds to the laser intensity amplitude of $1.67 \mathrm{TW} / \mathrm{cm}^{2}$ in the focal spot.

Under laser irradiation, the electron density in the laser channel increases up to $N_{e}=4.8 \cdot 10^{19} \mathrm{~cm}^{-3}$ (in Avogadro units $N_{e}=8 \cdot 10^{-5} \mathrm{Av}$ ) and doesn't exceed the critical one $N_{c}=10^{21} \mathrm{~cm}^{-3}\left(1.66 \cdot 10^{-3} \mathrm{Av}\right)$ for Nd:YAG laser light. Therefore, the laser radiation penetrates through the entire $\mathrm{Kr}$ layer depth damping exponentially. The electron temperature shown in Fig. 2c reaches $T_{e}=91 \mathrm{eV}$ near the upper $\mathrm{Kr}-\mathrm{He}$ interface of $\mathrm{Kr}$ layer to the time moment of $300 \mathrm{ps}$. It is higher than the ion temperature $T_{i}=58 \mathrm{eV}$, because the laser energy is absorbed by the plasma electrons. The average ion charge in krypton reaches a value of $\langle Z\rangle=23$ at the electron temperature maximum. The plasma is in non-equilibrium state; its emission is partially reabsorbed increasing the ionization degree significantly with respect to transparent plasma case [33].

The laser pulse power, laser absorption, spectrum integrated plasma emission and SXR emission in 2.887$3.234 \mathrm{~nm}$ waveband are represented in Fig. 3. A time integration of the emission power gives the following values: the krypton plasma absorbs of $345 \mathrm{~mJ}$ (or $69 \%$ of laser energy), the energy emitted in full spectrum is of $139 \mathrm{~mJ}$, the SXR energy emitted in a hard half of the water-window 2.47-3.234 nm waveband (below it will be mentioned as "hard water-window") is of 6 $\mathrm{mJ}$ (or $1.2 \%$ of laser energy), in a harder spectral band $0.207-2.47 \mathrm{~nm}$ it is of $0.9 \mathrm{~mJ}$ (or $0.18 \%$ of laser energy). In Fig. 4, a spatial distributions of plasma emittance in SXR radiation wavelength band $2.8867<\lambda<3.234$ $\mathrm{nm}$ are represented: (a) an instant value at a moment of peak emission $\mathrm{t}=300 \mathrm{ps}$ in $\mathrm{MW} / \mathrm{cm}^{3}$, (b) a timeintegrated value in $\mathrm{J} / \mathrm{cm}^{3}$. The time-integrated in-band SXR source is localized near the upper $\mathrm{Kr}-\mathrm{He}$ interface of $\mathrm{Kr}$ layer and looks like a solid hemisphere with diameter of $225 \mu \mathrm{m}$ FWHM. But the instant emission in this band is more like a shell of hemisphere of $250 \mu \mathrm{m}$ FWHM diameter. If to compare the image Fig. 4a of the instant emittance with the electron temperature distribution in Fig. 2c, one can see that the instant in-band $2.8867<\lambda<3.234 \mathrm{~nm}$ SXR emission at the maximal temperature and highest ionization degree is lower than in the "source shell" with electron 


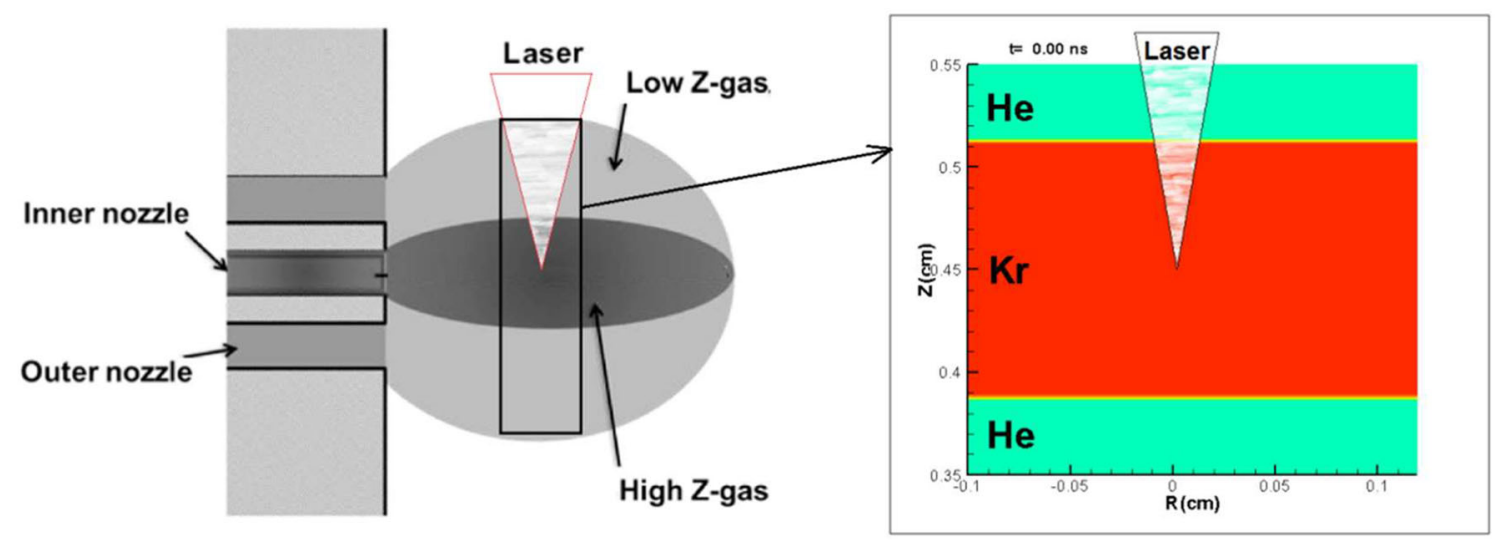

(a)

(b)

Fig. 1 a Typical schematic of double gas puff target irradiated with a laser and b zoomed simulated geometry of the central part of the double gas puff target with $(\mathrm{R}, \mathrm{Z})$ axes

temperature of $80 \div 81 \mathrm{eV}$ and average ion charge of $\langle\mathrm{Z}\rangle \approx 19.5 \div 20.5$, which is optimal [33] for the emission in the $2.8867<\lambda<3.234 \mathrm{~nm}$ waveband. That explains the instant shape of in-band SXR source, as the timeintegrated one includes a evolution of plasma through the optimal ionization state.

To the end of the laser pulse, the Kr plasma races a speed of spread under action of the increased plasma pressure in the opposite to the laser flux direction up to $v_{z}=1.0 \cdot 10^{6} \mathrm{~cm} / \mathrm{s}$ and in a radial direction up to $v_{r}=1.2 \cdot 10^{5} \mathrm{~cm} / \mathrm{s}$. This plasma spread reduces the plasma density and its temperature in a long time after SXR pulse from plasma. The longer wavelength plasma emission in UV and vacuum-UV continues over 3 ns duration (Fig. 3).

\subsubsection{Results on laser with energy $500 \mathrm{~mJ}$, pulse of 150 ps, intensity $2.45 \mathrm{TW} / \mathrm{cm}^{2}$}

The simulations results for an increased laser intensity are shown in Fig. 5, notably: (a) the plasma massdensity and (b) electron density distributions at 269 ps corresponding to the plasma SXR radiation peak for the laser pulse with energy $500 \mathrm{~mJ}$ and duration 150 ps FWHM focused in the focal spot of $330 \mu \mathrm{m}$ FWHM, which corresponds to the laser intensity amplitude in the focal spot $2.45 \mathrm{TW} / \mathrm{cm}^{2}$.

At given laser intensity in picosecond time range, the Kr plasma spreads under action of the increased plasma pressure in the opposite to the laser flux direction up to $v_{z}=1.5 \cdot 10^{6} \mathrm{~cm} / \mathrm{s}$ and in a radial direction up to $v_{r}=2.3 \cdot 10^{5} \mathrm{~cm} / \mathrm{s}$. The plasma ions do not have time to move away from the laser channel and an increase in the plasma electron density occurs against the background due to ionization in the laser radiation field.

Under laser irradiation, the electron density in the laser channel increases up to $N_{e}=5.4 \cdot 10^{19} \mathrm{~cm}^{-3}$ $\left(9 \cdot 10^{-5} \mathrm{Av}\right)$ and doesn't exceed the critical one $N_{c}=$ $10^{21} \mathrm{~cm}^{-3}\left(1.67 \cdot 10^{-3} \mathrm{Av}\right)$ for Nd:YAG laser light. Therefore, the laser radiation penetrates through the
Kr layer depth damping exponentially. The electron temperature represented in Fig. $5 \mathrm{c}$ reaches $T_{e}=175$ $\mathrm{eV}$ at $269 \mathrm{ps}$ and located near the upper $\mathrm{Kr}-\mathrm{He}$ interface of $\mathrm{Kr}$ layer. It exceeds the ion temperature $T_{i}=74$ $\mathrm{eV}$. The average ion charge of krypton reaches a value of $\langle\mathrm{Z}\rangle=26$ near the electron temperature maximum. The plasma is non-equilibrium; its emission is partially reabsorbed increasing the ionization degree significantly with respect to transparent plasma case [33].

The laser pulse power, laser absorption, spectrum integrated plasma emission and SXR emission in 2.887$3.234 \mathrm{~nm}$ waveband are represented in Fig. 6. A time integration of emission power gives: the krypton plasma absorbs of $361 \mathrm{~mJ}$ (or $72 \%$ of laser energy), the energy emitted in full spectrum is of $175 \mathrm{~mJ}$, the SXR energy emitted in $2.47-3.234 \mathrm{~nm}$ waveband is of $20.7 \mathrm{~mJ}$ (or $4.1 \%$ of laser energy), in a harder spectral band $0.207-$ $2.47 \mathrm{~nm}$ it makes of $5.6 \mathrm{~mJ}$ (or $1.1 \%$ of laser energy). Spatial distribution of plasma emittance in SXR radiation wavelength band $2.8867<\lambda<3.234 \mathrm{~nm}$ is represented in Fig. 7: (a) an instant value at a moment of emission maximum $\mathrm{t}=269 \mathrm{ps}$, (b) a time-integrated value. The time-integrated in-band SXR source is localized near the upper $\mathrm{Kr}-\mathrm{He}$ interface of $\mathrm{Kr}$ layer and looks like a shell of hemiellipsoid with diameter of $320 \mu \mathrm{m}$ FWHM prolate along Z-axis. The instant in band SXR emittance has a shape close to a hemiellipsoidal shell with the same diameter, but is thinner and not homogeneous, several periodical variations may be distinguished. If to compare the emittance in Fig. 7 to the electron temperature distribution in Fig. 5c, one can see that the instant in-band $2.8867<\lambda<3.234$ $\mathrm{nm}$ SXR emission at peak temperature and maximal ionization is lower than in the "shell-like source" with electron temperature of $80 \div 81 \mathrm{eV}$ and the average ion charge of $\langle\mathrm{Z}\rangle \approx 19.5 \div 20.5$, which is optimal for the emission in the $2.8867<\lambda<3.234 \mathrm{~nm}$ waveband. The variations in the instant emittance are produced during ionization instability of multicharged ion plasma in the intensive laser field, which is conditioned by strong 


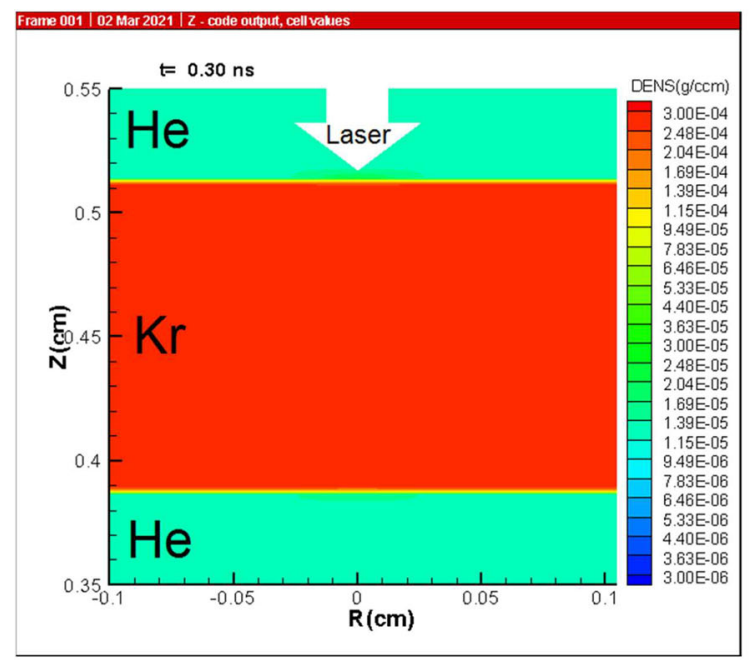

(a)

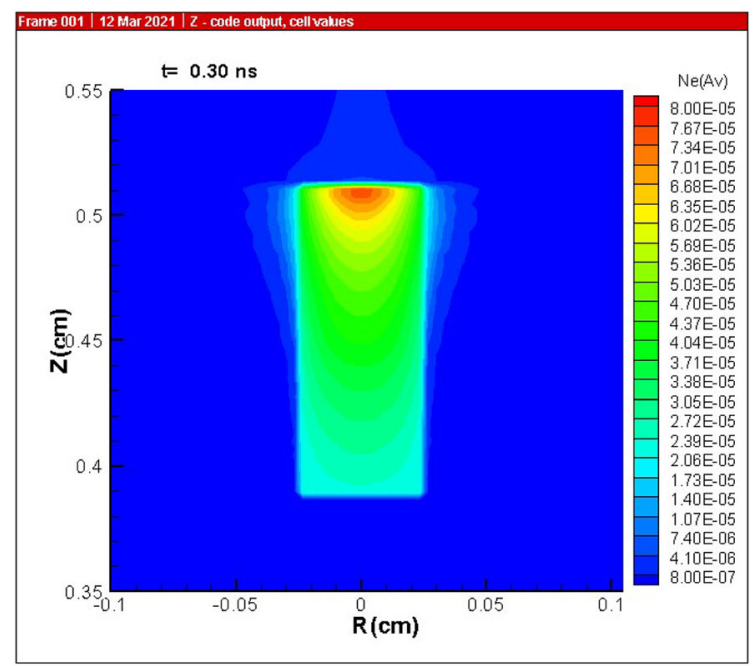

(b)

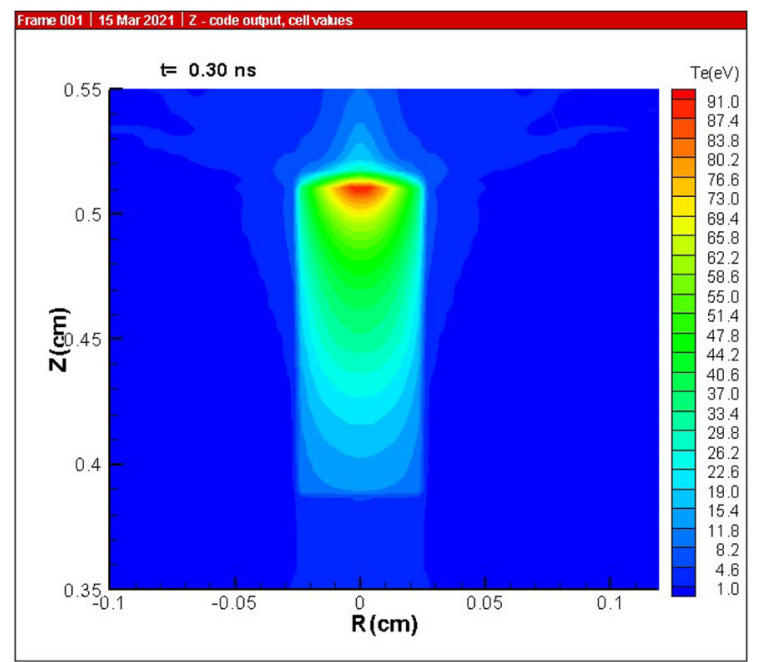

(c)

Fig. 2 Spatial distributions of plasma quantities: a massdensity in $\mathrm{g} / \mathrm{cm}^{3}, \mathrm{~b}$ electron density $N_{e}$ in Avogadro units $\left(\mathrm{Av}=6.0 \cdot 10^{23} \mathrm{~cm}^{-3}\right)$ and $\mathbf{c}$ electron temperature $T_{e}$ in $\mathrm{eV}$ at time-moment $300 \mathrm{ps}$ for $500 \mathrm{~mJ}: 150 \mathrm{ps}: 1.67 \mathrm{TW} / \mathrm{cm}^{2}$ laser pulse and at initial $\mathrm{Kr}$ gas mass density of $2.9 \cdot 10^{-4}$ $\mathrm{g} / \mathrm{cm}^{3}$

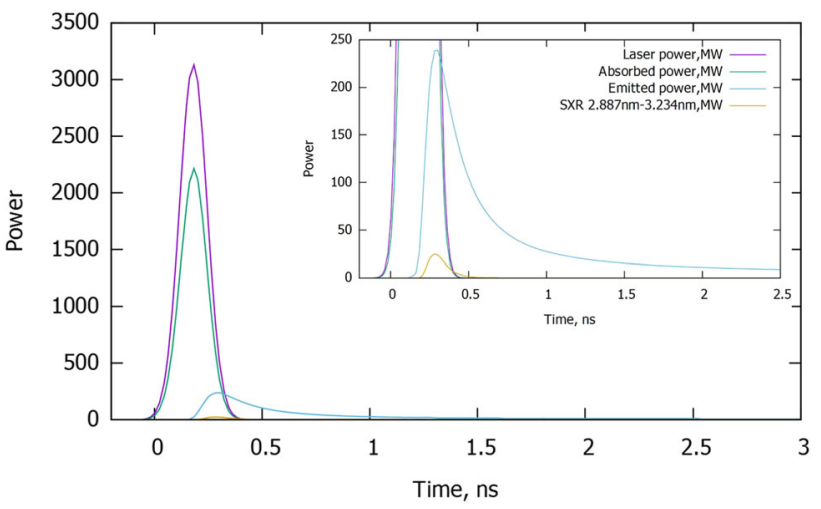

Fig. 3 Time dependencies of laser power, absorbed power by plasma, emitted spectrum integrated radiation power, emitted SXR radiation power in the spectral band $2.8867<$ $\lambda<3.234 \mathrm{~nm}$ for $500 \mathrm{~mJ}: 150 \mathrm{ps}: 1.67 \mathrm{TW} / \mathrm{cm}^{2}$ laser pulse

dependence of laser light collisional absorption (inversebremsstrahlung) on plasma ionization degree, e.g., in the case $\nu_{e i}^{2} ; \omega_{p e}^{2}<<\omega_{\text {las }}^{2}$

$$
\kappa \approx \frac{\omega_{p e}^{2}}{\omega_{\text {las }}^{2}} \frac{\nu_{e i}}{c} \sim \frac{\langle Z\rangle\left\langle Z^{2}\right\rangle}{T_{e}^{3 / 2}} N_{i}^{2}
$$

and of the plasma ionization degree on the plasma electron temperature $\left\langle Z^{2}\right\rangle \sim T_{e}^{\alpha}$, at $\alpha>5 / 4$ in certain range of the plasma temperatures. Also, an instability of Rayleigh-Taylor-type, initiated in formation of a divergent compression wave from the laser-discharge channel, may start to appear. The Rayleigh-Taylor instability is clearly manifested at nanosecond laser pulses, and its mechanism will be explained in detail below, during nanosecond laser plasma consideration. It should be said that the nor ionization instability, nor Rayleigh-Taylor instability was not manifested in previous smaller intensity laser pulse due to lower plasma pressure in the laser channel and twice less plasma spread speed. In the time-integrated shape of in-band SXR source the spots disappear, because it includes a complete plasma evolution through the optimal ionization and temperature instant. If to compare with the previous smaller intensity laser pulse, the temperature is much higher at increased laser intensity and the volume occupied by the plasma at optimal temperature of $80 \div 81 \mathrm{eV}$ for the in-band SXR emission is larger as well and consequently the in-band SXR emission multiplies in 4 times almost, exceeding $4 \%$ of the laser energy.

At the same time, the raised electron temperature at higher laser intensity provides a considerable contribution to the emission in harder radiation spectrum: it composes up to $1 \%$ of the laser energy in $0.2<\lambda<2.4$ nm waveband.

A further plasma spread lowers the plasma density and its temperature for a long time after SXR pulse generated in plasma. The longer wavelength plasma emission in UV, vacuum-UV lasts over 3 ns (see, Fig. 7). 


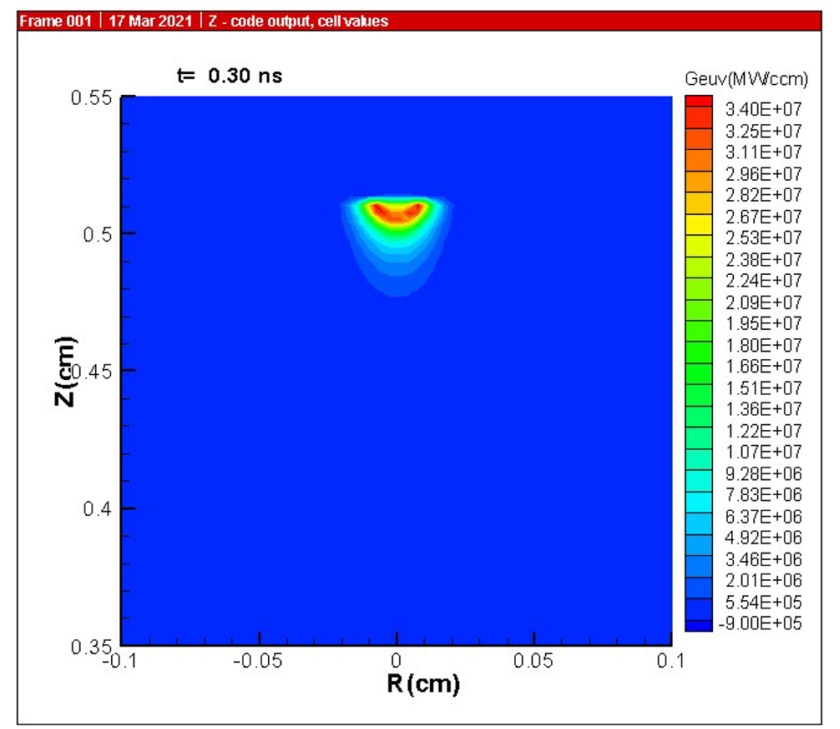

(a)

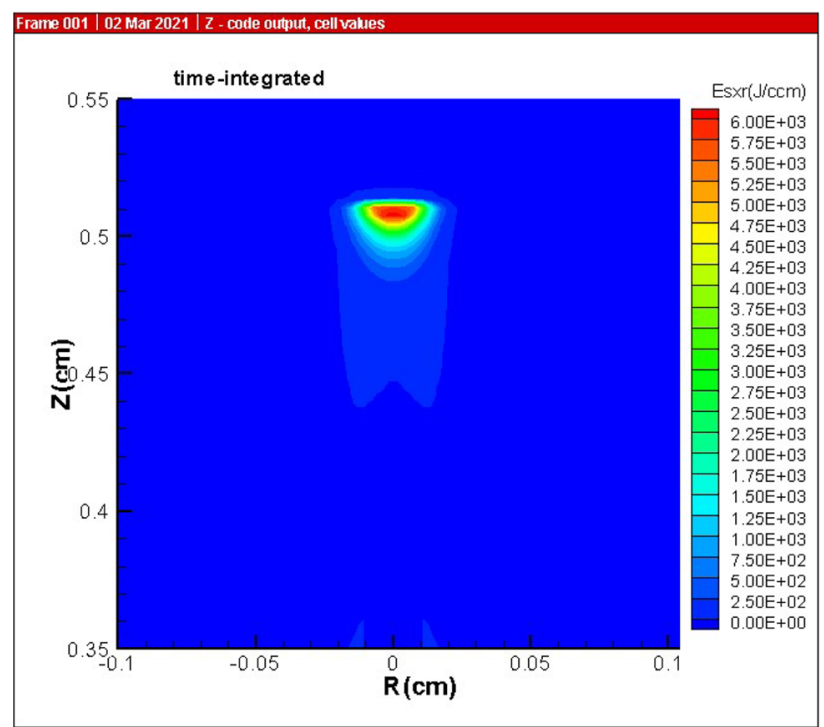

(b)

Fig. 4 Spatial distribution of emittance in SXR radiation wavelength band $2.8867<\lambda<3.234 \mathrm{~nm}$ : a an instant value at a moment of emission peak $\mathrm{t}=300 \mathrm{ps}$ in $\mathrm{MW} / \mathrm{cm}^{3}, \mathbf{b}$ a time-integrated value in $\mathrm{J} / \mathrm{cm}^{3}$ for $500 \mathrm{~mJ}: 150 \mathrm{ps}: 1.67$ $\mathrm{TW} / \mathrm{cm}^{2}$ laser pulse. The negative values correspond to the reabsorption

\subsection{Nanosecond laser plasma dynamics}

The incident laser beam of $\mathrm{TW} / \mathrm{cm}^{2}$ intensity causes the fast gas ionization due to the tunnel effect, an efficient acceleration of free electrons and a large rate of their collisions with atoms, which lead to a laser-plasma discharge development and a fast plasma formation. The laser light absorption increases self-consistently with ionization. The laser energy is absorbed in the krypton layer mainly. The plasma temperature and its pressure increase. On influence of the increasing plasma

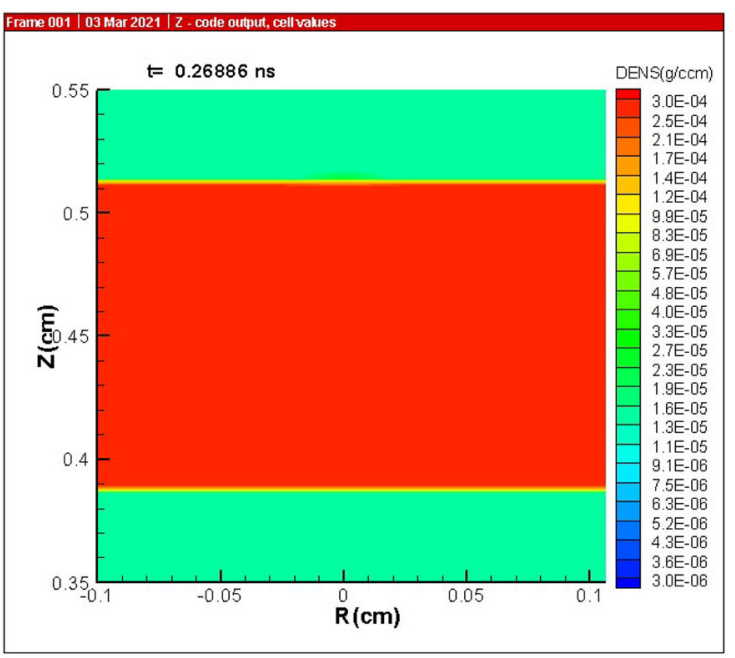

(a)

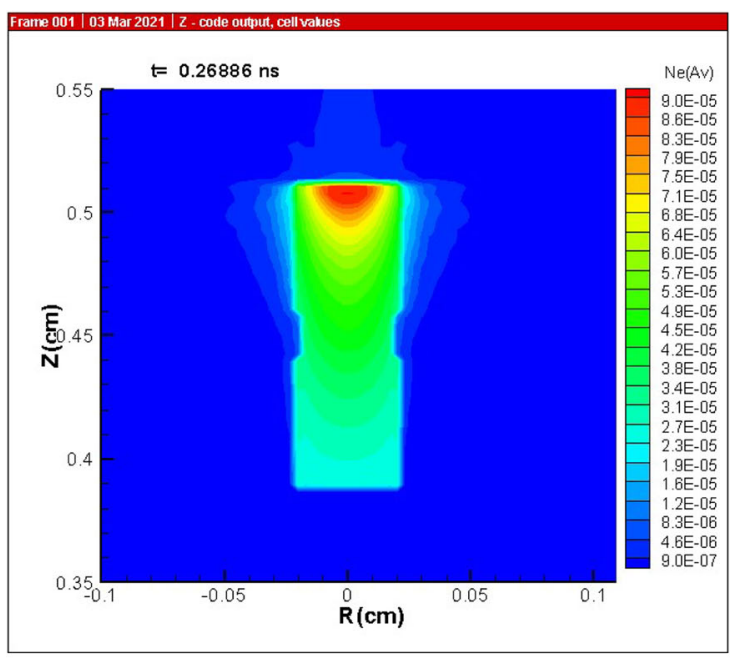

(b)

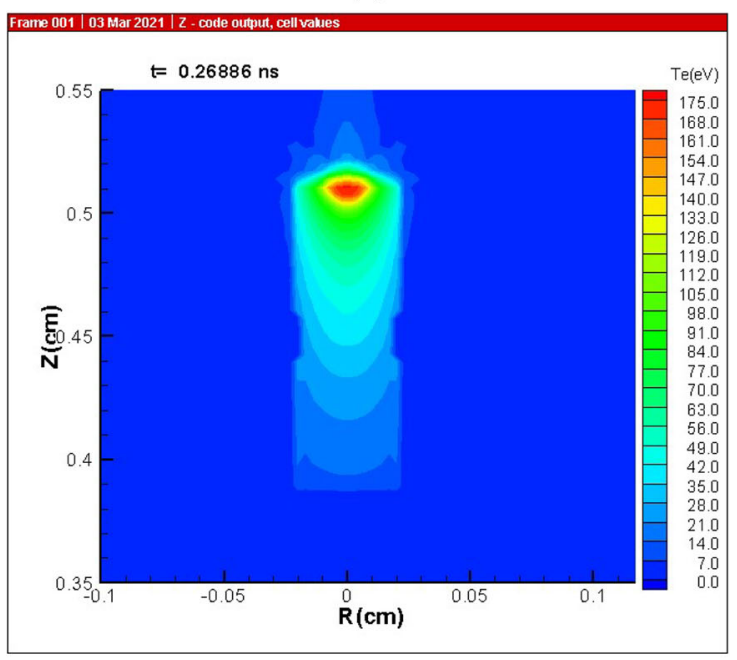

(c)

Fig. 5 Spatial distributions of plasma quantities: a massdensity in $\mathrm{g} / \mathrm{cm}^{3}, \mathbf{b}$ electron density $N_{e}$ in Avogadro units and c electron temperature $T_{e}$ in $\mathrm{eV}$ at time-moment 269 ps for $500 \mathrm{~mJ}: 150 \mathrm{ps}: 2.45 \mathrm{TW} / \mathrm{cm}^{2}$ laser pulse and at initial Kr gas mass density of $2.9 \cdot 10^{-4} \mathrm{~g} / \mathrm{cm}^{3}$ 


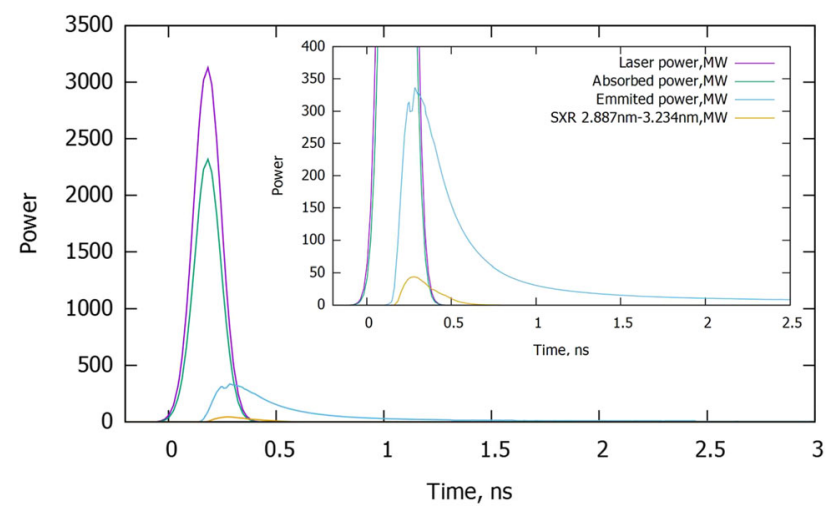

Fig. 6 Time dependencies of laser power, absorbed power by plasma, emitted spectrum integrated radiation power and emitted SXR radiation power in the wavelength band $2.8867<\lambda<3.234 \mathrm{~nm}$ for $500 \mathrm{~mJ}: 150 \mathrm{ps}: 2.45 \mathrm{TW} / \mathrm{cm}^{2}$ laser pulse

pressure the krypton plasma expands from the zone of laser absorption in the opposite to the laser flux direction mainly and in a radial direction. The plasma spread leads to the gas pushing out of the channel and the formation of a divergent compression wave transforming into the shock wave.

\subsubsection{Results on small LPP source with laser energy 0.6 $\mathrm{J}$, pulse $3 \mathrm{~ns}$, intensity $4.15 \mathrm{TW} / \mathrm{cm}^{2}$}

To the laser pulse intensity peak, the Kr plasma expands under action of the increased plasma pressure in the opposite to the laser flux direction up to $v_{z}=5.4 \cdot 10^{6} \mathrm{~cm} / \mathrm{s}$ and in radial direction up to $v_{r}=2.5 \cdot 10^{5} \mathrm{~cm} / \mathrm{s}$. The plasma spreads out of the laser channel and the formation of a divergent compression wave may be seen in Fig. 8a. In Fig. 8 are shown: (a) the plasma mass-density and (b) electron density distributions at $3.787 \mathrm{~ns}$ corresponding to the plasma SXR radiation maximum for the laser pulse of $600 \mathrm{~mJ}$ energy and duration 3ns FWHM focused in the focal spot of $63.2 \mu \mathrm{m}$ FWHM, which corresponds to the laser intensity amplitude in the focal spot $4.15 \mathrm{TW} / \mathrm{cm}^{2}$. In the compression wave, the $\mathrm{Kr}$ plasma mass density increases from initial $5.8 \cdot 10^{-4} \mathrm{~g} / \mathrm{cm}^{3}$ to $6.9 \div 7.8 \cdot 10^{-4} \mathrm{~g} / \mathrm{cm}^{3}$. In the laser channel plasma mass density drops down to $3 \cdot 10^{-4} \mathrm{~g} / \mathrm{cm}^{3}$.

Under laser irradiation, the electron density in the laser channel increases up to $N_{e}=1.26 \cdot 10^{19} \mathrm{~cm}^{-3}$ $\left(7.6 \cdot 10^{-5} \mathrm{Av}\right) 4$ and doesn't exceed the critical one $N_{c}=10^{21} \mathrm{~cm}^{-3}\left(1.66 \cdot 10^{-3} \mathrm{Av}\right)$ for Nd:YAG laser light. Therefore, the laser radiation penetrates through entire $\mathrm{Kr}$ layer depth damping exponentially. The electron temperature represented in Fig. $8 \mathrm{c}$ reaches $T_{e}=91$ $\mathrm{eV}$ at $3.787 \mathrm{~ns}$ near the upper $\mathrm{Kr}-\mathrm{He}$ interface in Kr layer. It excels the ion temperature $T_{i}=85 \mathrm{eV}$. The average ion charge of krypton reaches a value of $\langle\mathrm{Z}\rangle=22$ at the peak of electron temperature. The plasma is in non-equilibrium state, its emission is par-

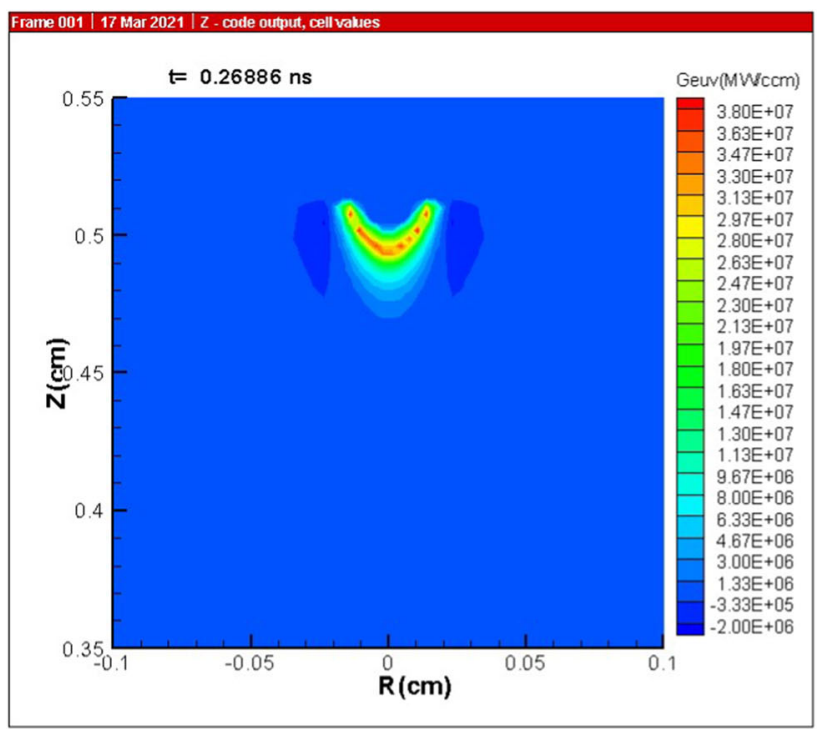

(a)

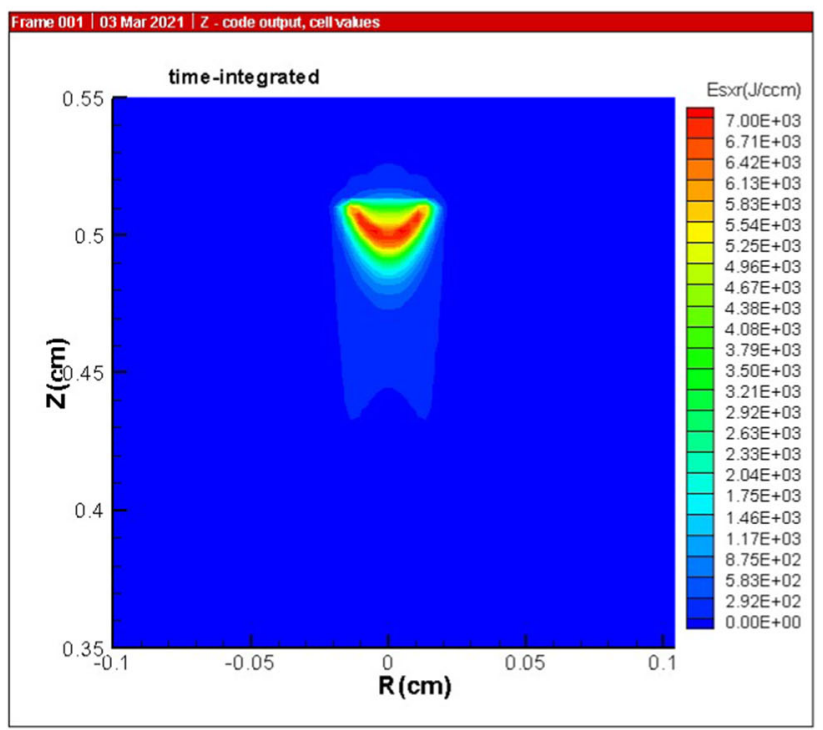

(b)

Fig. 7 Spatial distribution of emittance in SXR radiation wavelength band $2.8867<\lambda<3.234 \mathrm{~nm}$ : a an instant value at a peak of emission $\mathrm{t}=269 \mathrm{ps}$ in $\mathrm{MW} / \mathrm{cm}^{3}, \mathrm{~b}$ a time-integrated value in $\mathrm{J} / \mathrm{cm}^{3}$ for $500 \mathrm{~mJ}: 150 \mathrm{ps}: 2.45$ $\mathrm{TW} / \mathrm{cm}^{2}$ laser pulse. The negative values correspond to the reabsorption

tially reabsorbed increasing the ionization degree significantly with respect to transparent plasma case [33].

The laser pulse power, laser absorption, spectrum integrated plasma emission and SXR emission in 2.887$3.234 \mathrm{~nm}$ waveband are represented in Fig. 9. A time integration of emission power permits to obtain the following values: the krypton plasma absorbs of $0.47 \mathrm{~J}$ (or $78 \%$ of laser energy), the energy emitted in full spectrum is of $0.33 \mathrm{~J}$, the SXR energy emitted in $2.47-3.234$ $\mathrm{nm}$ waveband is of $36 \mathrm{~mJ}$ (or $6 \%$ of laser energy), the emitted energy in a harder spectral band 0.207-2.47 
$\mathrm{nm}$ is of $5.3 \mathrm{~mJ}$ (or $0.9 \%$ of laser energy). In Fig. 10, a spatial distribution of plasma emittance in SXR radiation wavelength band $2.8867<\lambda<3.234 \mathrm{~nm}$ is shown: (a) an instant value at a moment of peak emission at $\mathrm{t}=3.787 \mathrm{~ns}$, (b) a time-integrated value. The timeintegrated in-band SXR source is localized near the upper $\mathrm{Kr}-\mathrm{He}$ interface of $\mathrm{Kr}$ layer and looks like a solid ellipsoid with diameter of $125 \mu \mathrm{m}$ FWHM, prolate along $\mathrm{Z}$ axis with dimension of $163 \mu \mathrm{m}$. The instant emission in this band has a hemiellipsoidal shell with the same diameter and a little bit smaller dimension of $156 \mu \mathrm{m}$ in Z-axis. The instant in-band SXR emittance is not homogeneous, some variation in emittance may be distinguished between $32 \mathrm{TW} / \mathrm{cm}^{3}$ and $38 \mathrm{TW} / \mathrm{cm}^{3}$ with a slight change in electron temperature $(\sim 2 \%)$ and in plasma density as well $(\sim 1 \%)$. If to compare the image Fig. 10a of the instant emittance with electron temperature distribution in Fig. $8 \mathrm{c}$, one can see that the instant in-band $2.8867<\lambda<3.234 \mathrm{~nm}$ SXR emission at the peak temperature and the maximal ionization degree is lower than in the "source shell" with the electron temperature of $80 \div 81 \mathrm{eV}$ and average ion charge of $\langle Z\rangle \approx 19.5 \div 20.5$, which is optimal condition for the emission in $2.8867<\lambda<3.234 \mathrm{~nm}$ waveband. The presence of the variations in the instant emittance is conditioned by a development of Rayleigh-Taylortype instability, initiated in formation of a divergent compression wave from the laser-discharge channel. For given laser pulse, the instability is weak enough and produces just slight variation noted. This instability is clearly manifested at larger energy nanosecond laser pulses, and its mechanism will be explained in detail below in the $7.1 \mathrm{~J} / 1.1 \mathrm{~ns}$ laser pulse consideration. In the time-integrated shape of in-band SXR source, the spots overlay, because the integral includes a complete evolution of plasma through the optimal ionization and temperature instant.

If to compare the result with the previous case of less intensity picosecond laser, the mass density of plasma at optimal temperature of $80 \div 81 \mathrm{eV}$ is larger and emission duration for the in-band SXR is longer. So the in-band SXR emission grows, exceeding $6 \%$ of the laser energy. Considerable emission in harder radiation in $0.2<\lambda<$ $2.4 \mathrm{~nm}$ waveband is $0.9 \%$ of the laser energy.

The multigroup radiation transport approach used in RMHD Zstar provides the non-equilibrium selfconsistent with plasma state radiation features but with limited spectroscopic information on emission generated from plasma averaged in spectral groups without detailed resolution of spectra in lines. To analyze the detailed spectra and contribution of line emission into the water-window spectral region, the atomic structure of every ion with maximum amount of possible transitions between the excited states including single- and double-excited configurations has to be defined. Calculations of spectral emissivity for multicharged ions were done using the configuration interaction (CI) method [34] with 2 to 3 single-excited configurations considered together with the ground state configuration. The atomic levels and transition strengths were computed using Flexible Atomic Code (FAC) [35]. Resulted

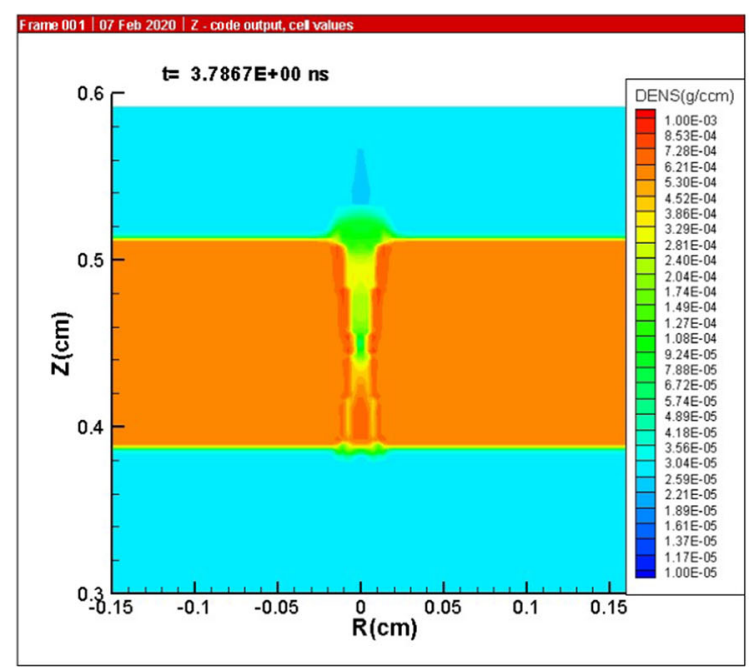

(a)

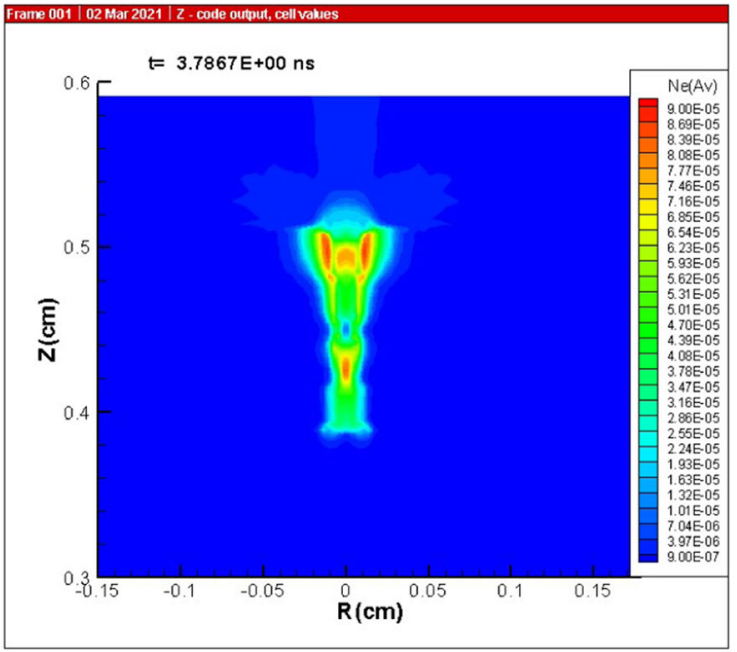

(b)

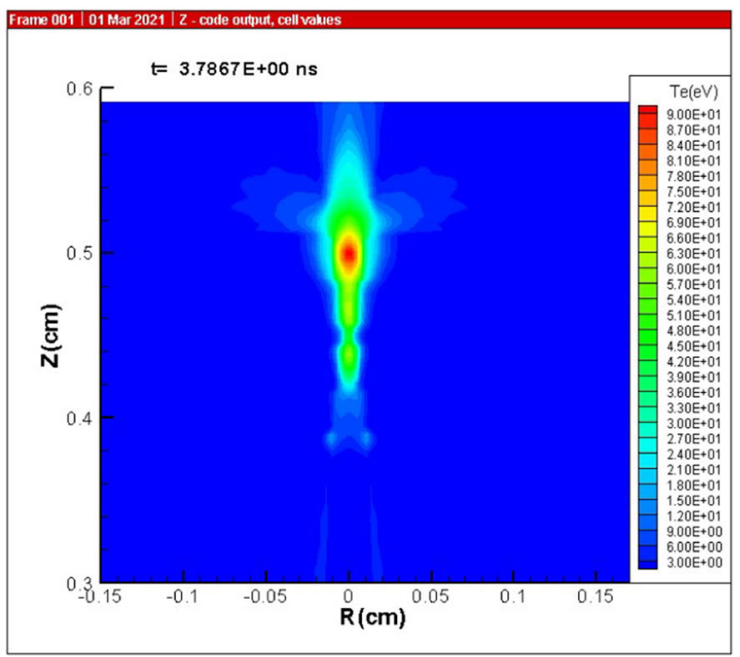

(c)

Fig. 8 Spatial distributions of plasma quantities: a massdensity in $\mathrm{g} / \mathrm{cm}^{3}, \mathbf{b}$ electron density $N_{e}$ in Avogadro units and (c) electron temperature $T_{e}$ in $\mathrm{eV}$ at $3.787 \mathrm{~ns}$ for 600 $\mathrm{mJ}: 3 \mathrm{~ns}: 4.15 \mathrm{TW} / \mathrm{cm}^{2}$ laser pulse and at initial $\mathrm{Kr}$ gas mass density of $5.8 \cdot 10^{-4} \mathrm{~g} / \mathrm{cm}^{3}$ 


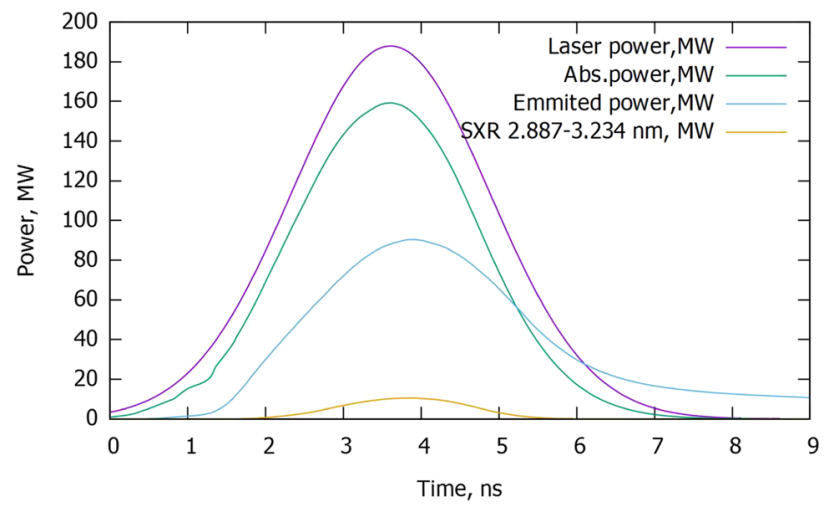

Fig. 9 Time dependencies of laser power, absorbed power by plasma, emitted spectrum integrated radiation power and emitted SXR radiation power in the wavelength band $2.8867<\lambda<3.234 \mathrm{~nm}$ for $600 \mathrm{~mJ}: 3 \mathrm{~ns}: 4.15 \mathrm{TW} / \mathrm{cm}^{2}$ laser pulse

atomic level energy structure and transition energies were corrected with the data obtained by THERMOS code based upon quantum-statistical HFS model [25]. Line emission spectra can be described by the spectral emissivity coefficient $j_{\omega}$ in bound-bound transitions, which includes level populations and oscillator's strengths of transitions $f_{\nu \mu}$

$$
j_{\omega}=\frac{e^{2} \hbar}{m_{e} c^{3}} \sum_{\nu, \mu} \omega^{3} N_{i} n_{\mu} \frac{g_{\nu}}{g_{\mu}} f_{\nu \mu} \phi_{\omega}
$$

Here, $\hbar \omega$ is the $\mu \rightarrow \nu$ transition energy; $N_{i}$ is the number of ions per unit volume; $n_{\mu}$ the relative population of the level $\mu ; g_{\nu}$ and $g_{\mu}$ are statistical weights of level $\nu$ and level $\mu$, respectively; $\varphi_{\omega}$ is a spectral line profile. The Gaussian profile was applied for spectral line broadening $\varphi_{\omega}$, that includes both Doppler and Stark effects.

Kr XIV-Kr XVIII lines contribute intensively to the water-window spectral region [33]. The satellite and resonant transitions ensure strong lines below $4 \mathrm{~nm}$. Due to the fact that the number of 3d-shell electrons drops with a greater ion charge, the number of states with lines acting in the water-window diminishes and the transition array narrows. In Kr XVIII ([Ar]3d) the 3p shell isn't completed. The transitions with the inner-shell electron $3 p$ (e.g., $3 \mathrm{p}^{6} 3 \mathrm{~d}-3 \mathrm{p}^{5} 3 \mathrm{~d} 4 \mathrm{~d}$ ) form a large array of lines in the $2-4 \mathrm{~nm}$ band. The resonant transitions $3 \mathrm{p}^{6} 3 \mathrm{~d}-$ $3 \mathrm{p}^{6} \mathrm{Xx}$ lie completely in the water-window range and it arrives to produce emission in the hard water-window range $\left(3 \mathrm{~d}^{1}-5 \mathrm{p}^{1}\right.$ is at around $\left.2.9 \mathrm{~nm}\right)$. The subsequent highly charged Kr XIX-Kr XXII ions and next have a number of strong lines situated below $3 \mathrm{~nm}$ in the hard water-window range, including the resonant transitions $3 \mathrm{p}^{n}-3 \mathrm{p}^{n-1} \mathrm{Xx}$. Since Kr XXI a considerable part of emission hits below $2 \mathrm{~nm}$ as well. From ions higher $\mathrm{Kr}$ XXIII the emission in the hard water-window range starts to diminish, they provide an emission in shorter spectral range. That is resumed in Fig. 11, presenting a resulting spectral emissivity for krypton ions consid-

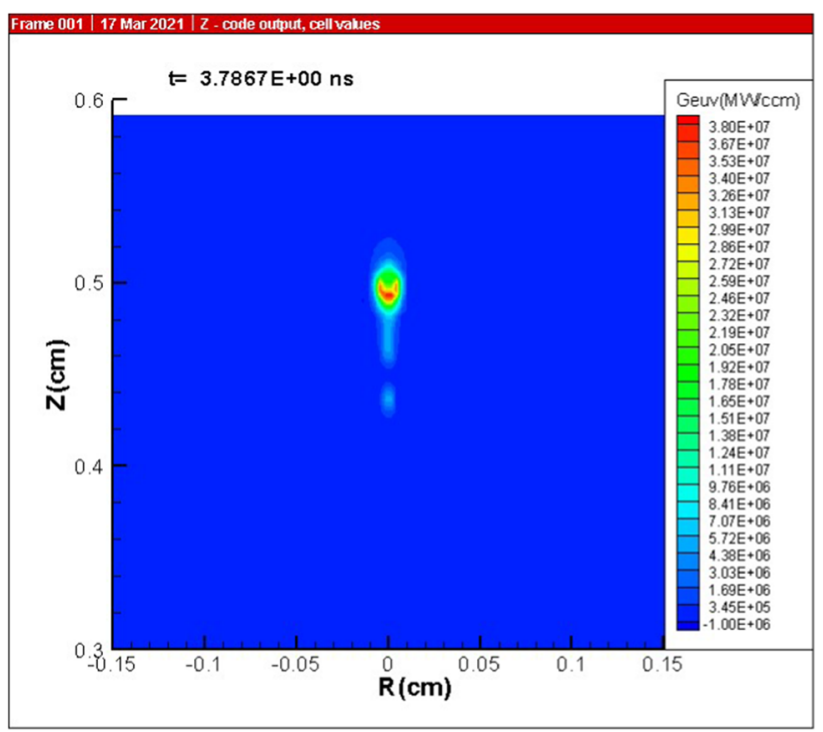

(a)

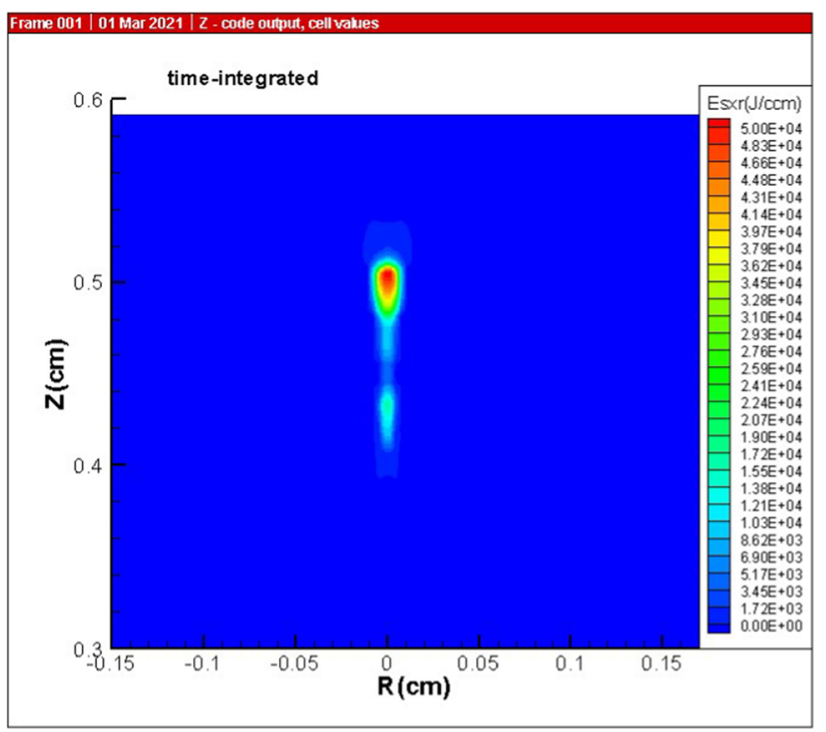

(b)

Fig. 10 Spatial distribution of emittance in SXR radiation wavelength band $2.8867<\lambda<3.234 \mathrm{~nm}$ : a an instant value at a moment of peak emission $\mathrm{t}=3.787 \mathrm{~ns}$ in $\mathrm{MW} / \mathrm{cm}^{3}, \mathbf{b}$ a time-integrated value in $\mathrm{J} / \mathrm{cm}^{3}$ for $600 \mathrm{~m} \mathrm{~J}: 3 \mathrm{~ns}: 4.15$ $\mathrm{TW} / \mathrm{cm}^{2}$ laser pulse. The negative values correspond to the reabsorption

erably contributing into hard water-window waveband $[32,33]$. The SXR radiation in $2.47-3.234 \mathrm{~nm}$ waveband is emitted from krypton plasma by ions from Kr XVIII to Kr XXIII.

To calculate a radiation transport in non-equilibrium plasma with tremendous amount of lines is not realistic yet. Therefore, a post-processing approach is applied for detailed emission spectrum treatment based on calculated detailed spectral properties described above and on the spectral escape factor calculated with multigroup radiation transport during RMHD simulation. 

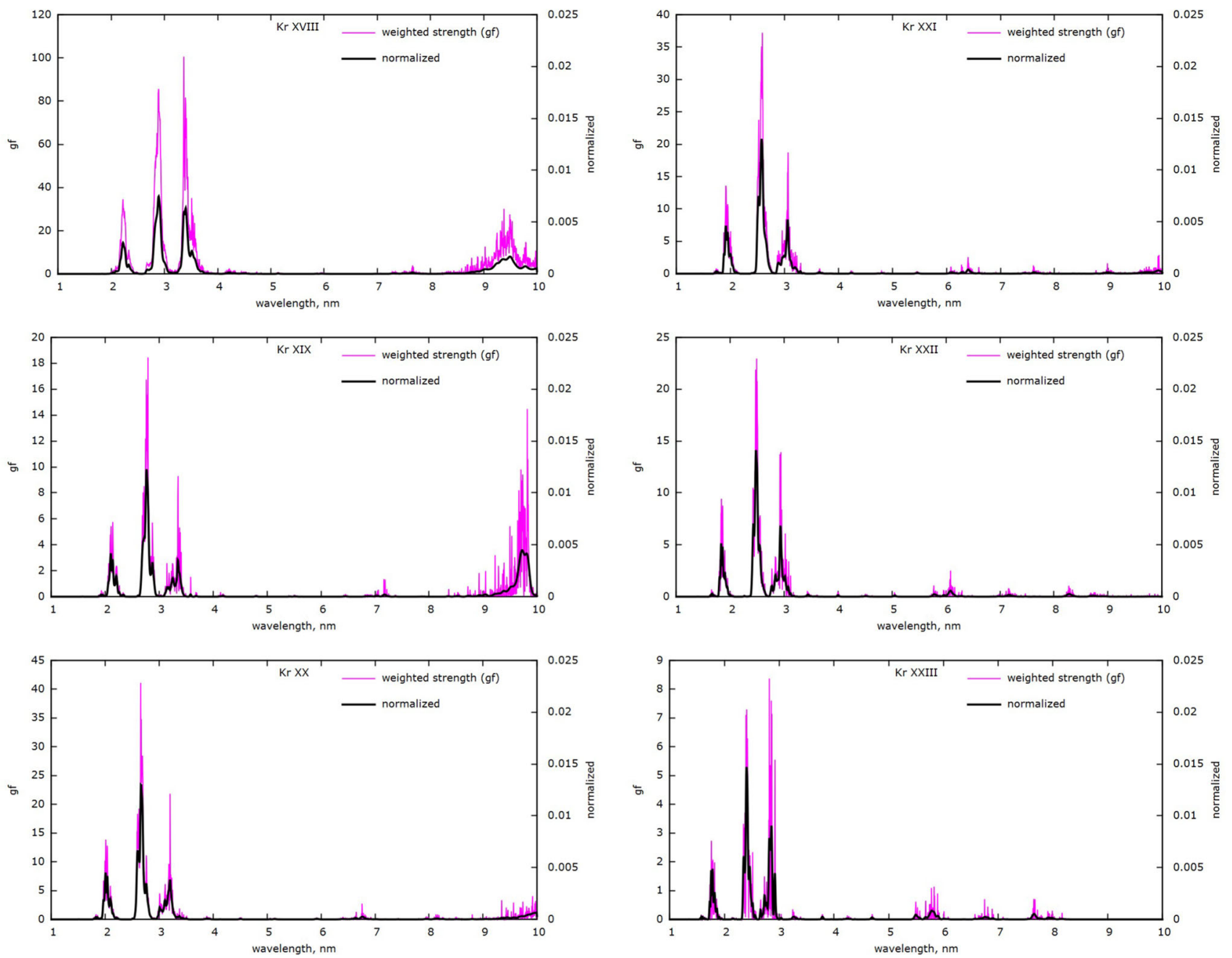

Fig. 11 Calculated weighted oscillator strengths in krypton ions Kr XVIII-Kr XXIII intensively contributing into hard water-window waveband and their normalized profile (please note that the ranges for gf and for normalized values are not equal)

It uses data on spatial-temporal plasma parameters distributions (distribution of plasma density, temperatures, etc.) from Zstar-code simulation data to calculate an emission capability of plasma. An example of the instant entire plasma volume emission capability calculated at different time instants is represented in Fig. 12a.

The spectral escape factor is calculated as

$$
q_{\omega}(t)=\frac{\int_{V} G_{\omega} d V}{4 \pi \int_{V} j_{\omega} d V}
$$

where $G_{\omega}$ is the specific spectral radiation power (emittance), e.g., the calculated one is presented in Fig. 10, particularly for the waveband of $2.8867<\lambda<3.234$ $\mathrm{nm} ; j_{\omega}$ is the specific spectral emission capability (emissivity) of the substance into a unit of solid angle (in the code Zstar it is approximated from atomic tables calculated in pre-processing). The spectral escape factor characterizes a fraction of radiation at frequency $\omega$ escaping of the volume $V$. It permits to estimate the detailed output spectrum from known $j_{\omega}$ without explicit integration of the radiation transport equation. Then, the calculated instant entire plasma volume emission capability (see, Fig. 12) with addition of a continuum spectral part is multiplied by the spectral escape factor to obtain the detailed spectral emission power in the waveband desired. A time integrated detailed spectral output for simulated LPP with given parameters is represented in Fig. 12b.

\subsection{Results on large LPP source with laser energy $7.1 \mathrm{~J}$, pulse $1.1 \mathrm{~ns}$, intensity $3.34 \mathrm{TW} / \mathrm{cm}^{2}$}

The laser energy is absorbed in the krypton layer mainly. The plasma temperature and its pressure increase. To the end of the laser pulse, the Kr expands under the impact of the increased plasma pressure in the opposite to the laser flux direction with the velocity up to $v_{z}=1.5 \cdot 10^{7} \mathrm{~cm} / \mathrm{s}$ and in a radial direction 


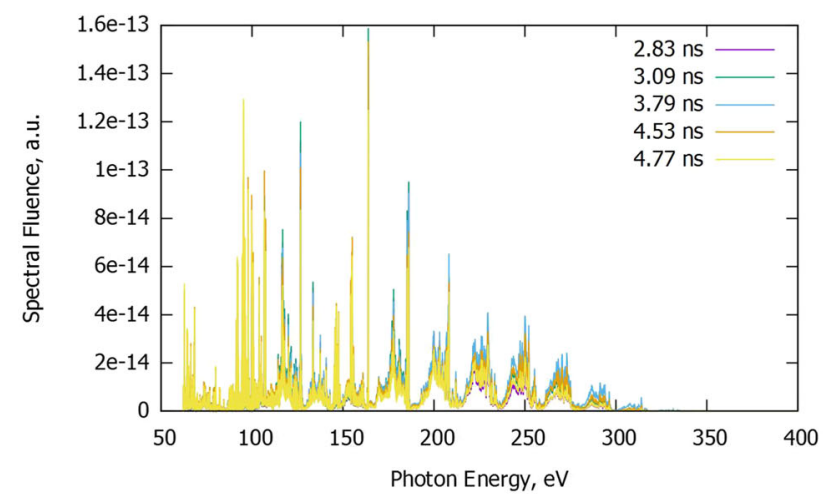

(a)

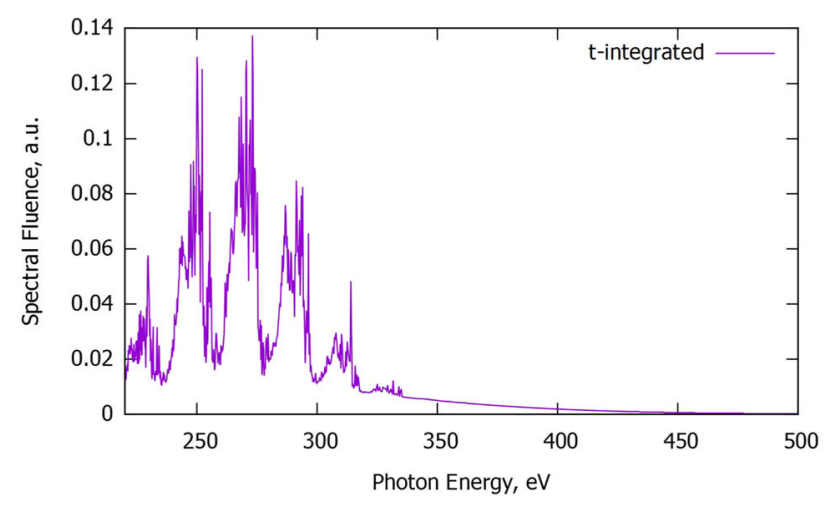

(b)

Fig. 12 Resulting spectral data He:Kr double gas puff simulation from entire plasma volume for $600 \mathrm{~mJ}$ : $3 \mathrm{~ns}$ : $4.15 \mathrm{TW} / \mathrm{cm}^{2}$ laser pulse: a normalized instant detailed line emission capability in EUV/SXR range at different timemoments from 2.83 to $4.77 \mathrm{~ns}$; b time-integrated detailed emission spectrum weighted with the spectral escape factor in $230 \div 500 \mathrm{eV}$ spectral range

with the velocity up to $v_{r}=7.4 \cdot 10^{6} \mathrm{~cm} / \mathrm{s}$, which considerably exceeds the sound speed and a strong divergent shock wave forms.

In Fig. 13, the results of plasma modeling at $t=1.98$ ns are shown, corresponding to the peak of plasma SXR radiation for the laser pulse of $7.1 \mathrm{~J}$ energy and duration $1.1 \mathrm{~ns}$ FWHM focused in the focal spot of $400 \mu \mathrm{m}$ FWHM, which matches to the laser intensity amplitude in the focal spot of $3.34 \mathrm{TW} / \mathrm{cm}^{2}$ : (a) the plasma massdensity and (b) electron density distributions and (c) the electron temperature. The electron density doesn't exceed the critical one $N_{c}=10^{21} \mathrm{~cm}^{-3}\left(1.67 \cdot 10^{-3} \mathrm{Av}\right)$ for Nd:YAG laser light. Therefore, the laser radiation penetrates through the entire $\mathrm{Kr}$ layer depth damping exponentially. In Fig. 13b, one may observe a secondary ionization of the krypton plasma outside the laser channel by SXR plasma emission from the central zone and its reabsorption in surrounding cold gas.

The electron temperature represented in Fig. 13c reaches $T_{e}=306 \mathrm{eV}$ in the laser channel to the time moment of $t=1.98 \mathrm{~ns}$ in the region located near the upper $\mathrm{Kr}-\mathrm{He}$ interface of $\mathrm{Kr}$ layer. It overcomes the ion temperature $T_{i}=296 \mathrm{eV}$ due to laser heating. The average ion charge of krypton reaches a value of $\langle Z\rangle=29$ near the peak of electron temperature. The plasma is in non-equilibrium state, its emission is partially reabsorbed increasing the ionization degree significantly with respect to transparent plasma case [33].

Heating with laser light, ionization and consequent increase in plasma pressure leads to expansion of the plasma from the laser absorption channel and formation of a divergent compression wave transforming further to a shock wave. Behind the shock wave front, the ion temperature of krypton plasma is higher than the electron one due to the viscous heating of ions in the shock wave primary.

The laser pulse power, laser absorption, spectrum integrated plasma emission and SXR emission in 2.887 $3.234 \mathrm{~nm}$ waveband are represented in Fig. 14. The emission power integration over a time gives: the krypton plasma absorbs of $5.49 \mathrm{~J}$ (or $77 \%$ of laser energy), the energy emitted in full spectrum is of $4.34 \mathrm{~J}$, the SXR energy emitted in 2.47-3.234 nm waveband is of $0.62 \mathrm{~J}$ (or $8.7 \%$ of laser energy), and in a harder spectral band $0.207-2.47 \mathrm{~nm}$ the emitted energy reaches $1.22 \mathrm{~J}$ (or 17\% of laser energy). Three main peaks and irregular vibrations are observed in SXR emission power profile. Spatial distribution of SXR plasma emittance in wavelength band of $2.8867<\lambda<3.234 \mathrm{~nm}$ at three peaks together with the time-integrated are represented in Fig. 15: (a) an instant value at three consequent time moments $\mathrm{t}=0.86 \mathrm{~ns}, \mathrm{t}=1.98 \mathrm{~ns}$ (SXR emission maximum) and $\mathrm{t}=2.99 \mathrm{~ns}$ in $\mathrm{MW} / \mathrm{cm}^{3}$; (b) a time-integrated value in $\mathrm{J} / \mathrm{cm}^{3}$ for $7.1 \mathrm{~J}: 1.1 \mathrm{~ns}: 3.34$ $\mathrm{TW} / \mathrm{cm}^{2}$ laser pulse. The negative values correspond to where the SXR reabsorption predominates over the SXR emission. The time-integrated in-band SXR source is localized near the upper $\mathrm{Kr}-\mathrm{He}$ interface of $\mathrm{Kr}$ layer and looks like an inhomogeneous shell of hemisphere with diameter of $780 \mu \mathrm{m}$ FWHM slightly oblate along Z-axis, consisting of spots. The instant emission in this band has an inhomogeneous hemisphere shell profile with diameter of $680 \mu \mathrm{m}$ FWHM slightly oblate along $\mathrm{Z}$ axis, consisting of spots also. If to compare Fig. 15 of the emittance to electron temperature distribution in Fig. 13c, one can see that the instant SXR emission in-band $2.8867<\lambda<3.234 \mathrm{~nm}$ at a peak of temperature and ionization is less then in the "shelllike source" with the electron temperature of $87 \div 92$ $\mathrm{eV}$ and the average ion charge of $\langle\mathrm{Z}\rangle \approx 19.5 \div 20.5$, which is optimal for the emission in such waveband. These conditions are achieved just behind the shock wave front, where Kr plasma mass-density increases up to $\rho=8.7 \cdot 10^{-4} \mathrm{~g} / \mathrm{cm}^{3}$ and the electron density is up to $\mathrm{N}_{e}=1.3 \cdot 10^{20} \mathrm{~cm}^{-3}\left(2.1 \cdot 10^{-4} \mathrm{Av}\right)$. Kr plasma ionization equilibrium for optimal charge is shifted to higher temperatures with respect to previous ps and ns cases because of greater plasma density. In that zone behind the shock wave front, the ion temperature of krypton plasma is $T_{i}=103 \div 108 \mathrm{eV}$. A significant excess of the ion temperature over the electron one is a characteristic feature of the shock wave. The SXR emission is localized exactly behind the divergent shock wave front and 
expands with it. The most optimal ionization degree for SXR emission in $2.8867<\lambda<3.234 \mathrm{~nm}$ waveband together with large enough volume occupied by emitting plasma is realized at $\mathrm{t}=1.98 \mathrm{~ns}$ corresponding to the maximum of SXR emittance.

The presence of bright spots in the instant and timeintegral emittance is conditioned by a development of Rayleigh-Taylor-type instability, initiated in formation of a divergent compression wave from the laserdischarge channel. Heating with laser light, ionization and consequently increase in plasma pressure leads to expansion of the plasma from the laser absorption channel and formation of a divergent compression wave transforming to a shock wave. At the compression wave front, the plasma density rises, and decreases behind the front, although the plasma pressure maximizes in the absorption zone of laser light behind the compression wave front. Thus, a state occurs when the pressure gradients and plasma density are oppositely directed, i.e., $\nabla \mathrm{P} \cdot \nabla \rho<0$. This condition corresponds to instability criterion of the Rayleigh-Taylor type instability [36]. A typical distribution of plasma density and temperature of the krypton target under laser irradiation is presented in Fig. 16. The instability leads to the redistribution of plasma density and the formation of spots of increased SXR emission behind the compression wave front. The development of this instability may be seen also in Fig. 14 as irregular vibrations of the spectrum integrated emission power and of SXR one.

If to compare with the previous smaller intensity laser pulse, the temperature is getting much higher, the volume occupied by the plasma at optimal temperature for the in-band SXR emission in 2.47-3.234 $\mathrm{nm}$ waveband is larger and consequently the in-band SXR emission is much more intensive, exceeding $8.7 \%$ of the laser energy. At the same time, the increased electron temperature at higher laser intensity provides significant emission in harder part of the spectrum: in $0.2<\lambda<2.4 \mathrm{~nm}$ waveband it is up to $17 \%$ of the laser energy. The plasma spread will reduce the plasma density and its temperature for a long time after SXR pulse from plasma. The emission from plasma in UV and vacuum UV scopes lasts for over $10 \mathrm{~ns}$ (Fig. 15).

\section{Conclusions}

The self-consistent computer modeling of laser multicharged ion plasma, induced in double stream gas puff with high-Z krypton stream in an annular low-Z helium jet as a circumferential gas as a source of soft X-ray radiation, is performed with RMHD Zstar code and the obtained results are analyzed in detail. Dynamics of plasma produced by Nd:YAG laser beam of picosecond and nanosecond pulse duration in $\mathrm{TW} / \mathrm{cm}^{2}$ laser intensity range with various energies and focusing parameters, concordant to the experimental setups, and the emission generation processes in the plasma are investigated.

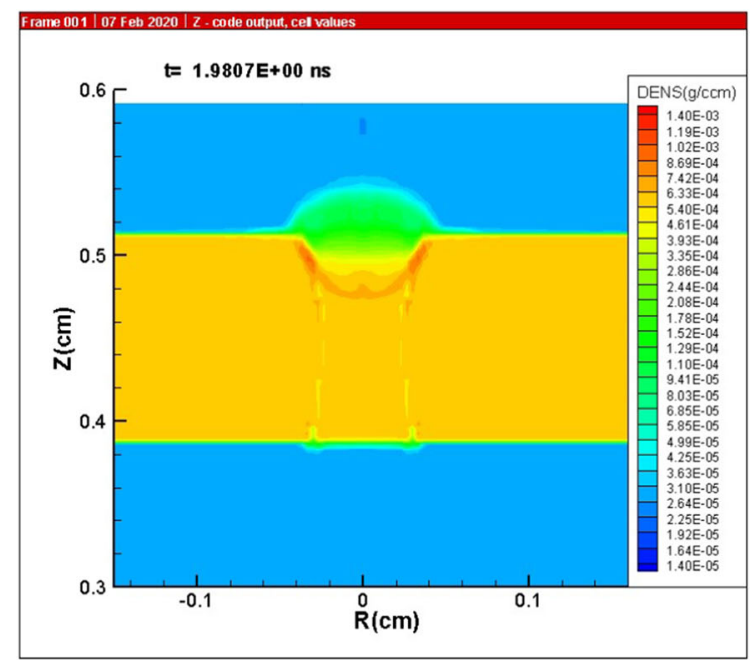

(a)

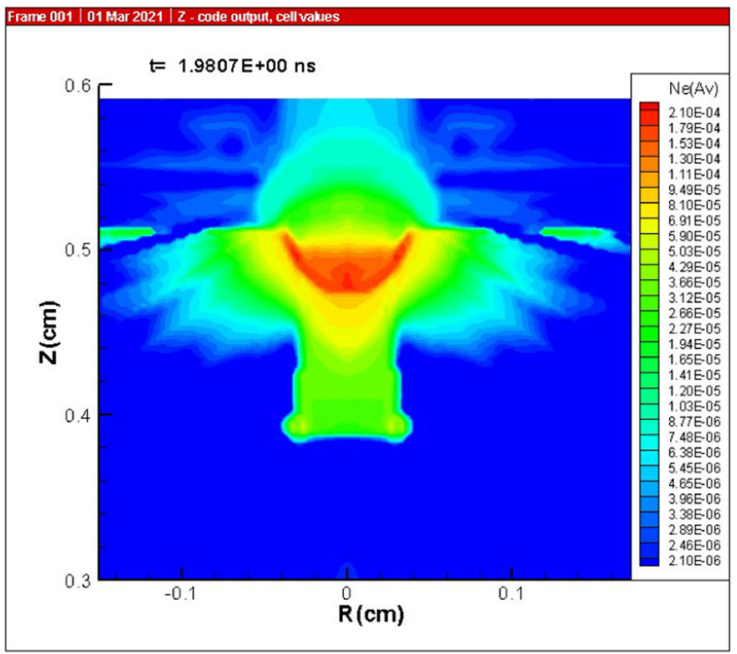

(b)

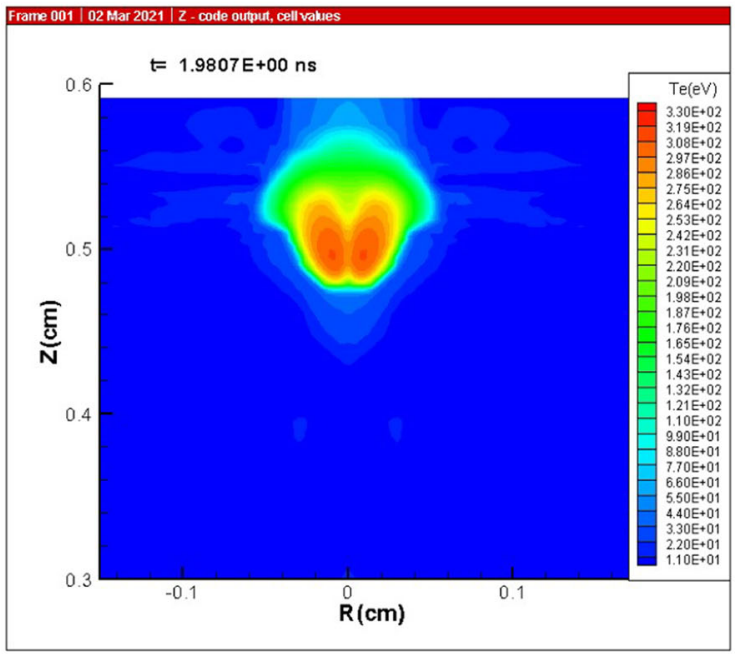

(c)

Fig. 13 Spatial distributions of plasma quantities: a massdensity in $\mathrm{g} / \mathrm{cm}^{3}$, b electron density $N_{e}$ in Avogadro units and $\mathbf{c}$ electron temperature $T_{e}$ in $\mathrm{eV}$ at $1.98 \mathrm{~ns}$ for $7.1 \mathrm{~J}$ : $1.1 \mathrm{~ns}: 3.34 \mathrm{TW} / \mathrm{cm}^{2}$ laser pulse and at initial $\mathrm{Kr}$ gas mass density of $5.8 \cdot 10^{-4} \mathrm{~g} / \mathrm{cm}^{3}$ 


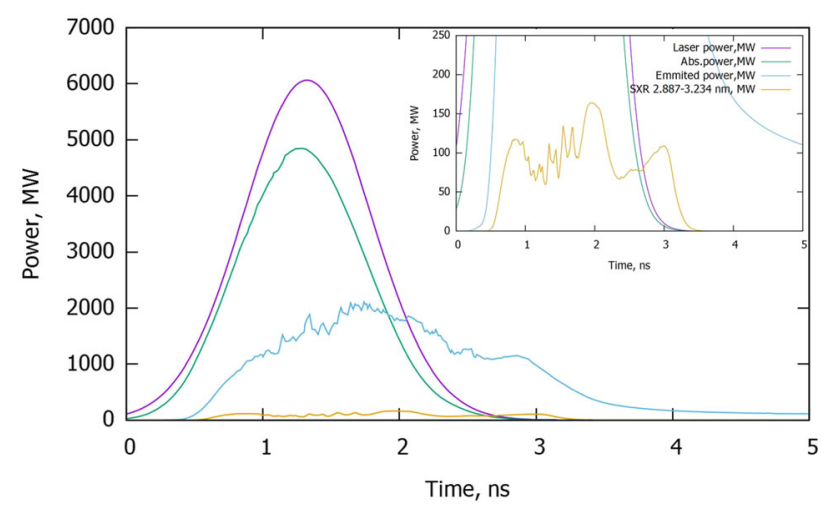

Fig. 14 Time dependencies of laser power, absorbed power by plasma, emitted spectrum integrated radiation power and emitted SXR radiation power in the wavelength band $2.8867<\lambda<3.234 \mathrm{~nm}$ for $7.1 \mathrm{~J}: 1.1 \mathrm{~ns}: 3.34 \mathrm{TW} / \mathrm{cm}^{2}$ laser pulse

The incident laser beam of $\mathrm{TW} / \mathrm{cm}^{2}$ intensity causes the fast gas ionization due to the tunnel effect, the efficient acceleration of free electrons and their collisions with atoms, which lead to a laser-plasma discharge development and fast plasma formation. The laser light absorption increases self-consistently with ionization. The laser energy is absorbed in the krypton layer mainly from the upper $\mathrm{Kr}-\mathrm{He}$ interface damping exponentially. The plasma temperature and its pressure increase. The picosecond and nanosecond laser beam interaction with double-gas puff target are very different. For a ps pulse, gas is rapidly ionized in the laser beam channel, but the gas does not have time to shift enough during the pulse, and the plasma electron density grows against the background of almost constant ion density during the ionization in the laser radiation field. At the ns pulse, the ionization occurs and the gas heating leads to the gas pushing out of the channel and to the formation of a divergent compression wave transforming into the shock wave. Behind the compression wave front, conditions arise for the development of Rayleigh-Taylor-type instability. The instability leads to the redistribution of plasma temperature and electron density, and the formation of spots of increased soft X-ray emission. The developed plasma is in a far from the local thermodynamic equilibrium state: there is no equilibrium between plasma and radiation and between electrons and ions in plasma due to transient effects in multicharged ion plasma. The fundamental understanding of these is a key to efficient optimization of a plasma radiation source. The plasma is semitransparent for the self-emission. The peak value of electron temperature is higher than the value of ion temperature in laser channel during the laser pulse. But the ion temperature exceeds the electron one behind the divergent shock wave front. The physical mechanisms of SXR radiative zone formation are different as well for picosecond and nanosecond pulses, and for low and high energy lasers. For a ps pulse or low energy ns pulse, the optimal conditions of ionization state (partic-
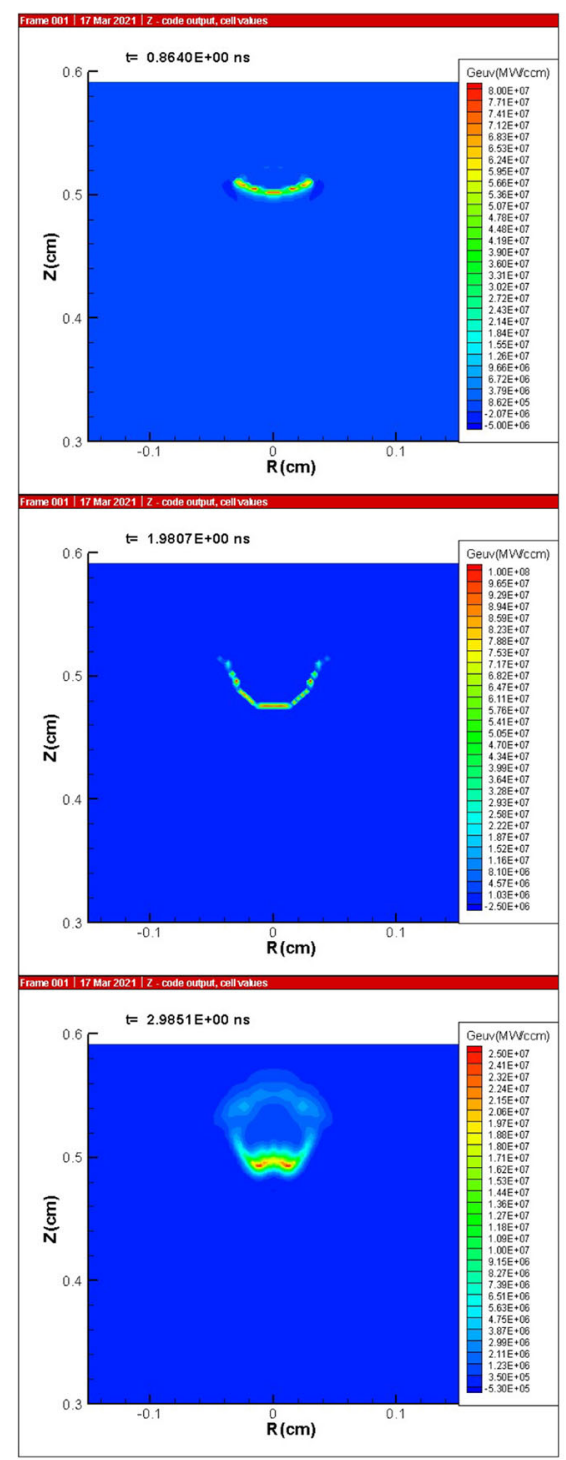

(a)

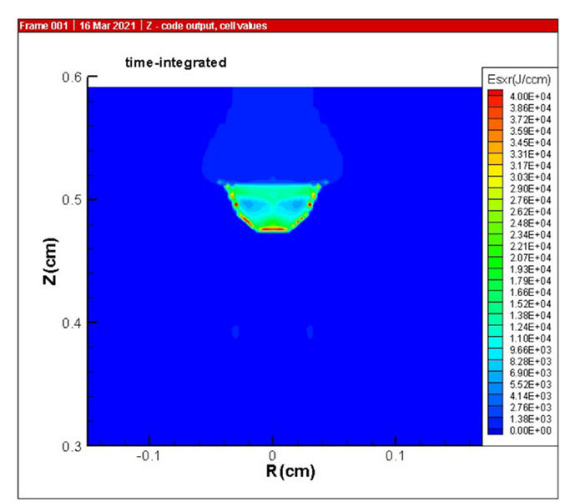

(b)

Fig. 15 Spatial distribution of emittance in SXR radiation wavelength band $2.8867<\lambda<3.234 \mathrm{~nm}$ : a an instant value at three consequent time moments: $\mathrm{t}=0.86 \mathrm{~ns}, \mathrm{t}=1.98$ $\mathrm{ns}\left(\mathrm{SXR}\right.$ emission peak) and $\mathrm{t}=2.99 \mathrm{~ns}$ in $\mathrm{MW} / \mathrm{cm}^{3} ; \mathbf{b}$ a time-integrated value in $\mathrm{J} / \mathrm{cm}^{3}$ for $7.1 \mathrm{~J}: 1.1 \mathrm{~ns}: 3.34$ $\mathrm{TW} / \mathrm{cm}^{2}$ laser pulse. The negative values correspond to the SXR reabsorption 


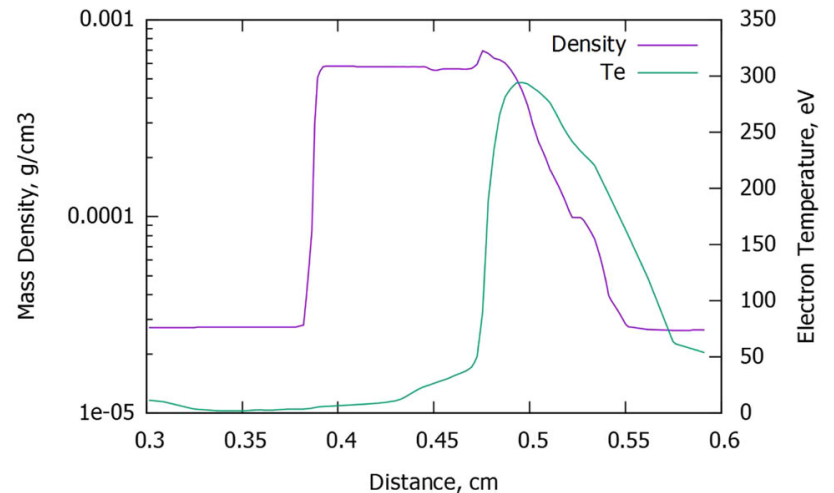

Fig. 16 Calculated profiles of plasma mass-density (magenta line, left-hand side log scale axis) and electron temperature (green line, right-hand side linear axis) vs. distance along Zaxis at $R=0$, at a time $1.98 \mathrm{~ns}$ for $7.1 \mathrm{~J}$ : $1.1 \mathrm{~ns}: 3.34 \mathrm{TW} / \mathrm{cm}^{2}$ laser pulse

ularly for hard water-window emission from krypton it is $\langle\mathrm{Z}\rangle \approx 19.5 \div 20.5$ ) are attained in the laser channel by means of direct laser heating. At a high energy (of few Joules) ns pulses, the plasma in the laser channel is rarefied due to its spread, and is overheated. The optimal conditions of ionization state and high enough plasma density are achieved behind the divergent shock wave. But the hardest by spectrum radiation is emitted from the hottest zone, which is located inside the plasma channel. The conversion efficiency of laser energy to SXR emission in $2.47<\lambda<3.234 \mathrm{~nm}$ waveband inside the water-window range at low laser energy (of $500 \div 600 \mathrm{~mJ}$ ) increases with the laser intensity (focusing degree) from $1.2 \%$ (for ps pulse, $1.67 \mathrm{TW} / \mathrm{cm}^{2}$ ) and $4.1 \%$ (for ps pulse, $2.45 \mathrm{TW} / \mathrm{cm}^{2}$ ) to $6 \%$ (for ns pulse, $4.15 \mathrm{TW} / \mathrm{cm}^{2}$ ), and up to $8.7 \%$ for large energy ns laser pulse of $3.34 \mathrm{TW} / \mathrm{cm}^{2}$ intensity. The SXR emission of double gas puff target increases with laser energy in spite of even less laser intensity, i.e., a ns pulse is more effective rather than ps one at optimal conditions of double gas puff target.

An intensive radiation in a harder part of emission spectrum, notably in $0.2<\lambda<2.4 \mathrm{~nm}$ waveband, making up to $17 \%$ of the laser energy can be obtained at high laser energy only, that corresponds to the experimental results of the group at Institute of Optoelectronics MUT in Warsaw [32].

Acknowledgements The authors would like to thank Prof H. Fiedorowicz and Dr P.W. Wachulak from Institute of Optoelectronics, Military University of Technology in Warsaw for the fruitful collaboration and discussions. This article is based upon work from COST Action TUMIEE (CA17126), supported by COST (European Cooperation in Science and Technology).

Data Availability Statement This manuscript has no associated data or the data will not be deposited. [Authors' comment: The data cannot be deposited because of its specific format, which is not acceptable by depository resources.
To obtain the data and its format description, the interested person is welcome to contact the authors directly by email.]

Open Access This article is licensed under a Creative Commons Attribution 4.0 International License, which permits use, sharing, adaptation, distribution and reproduction in any medium or format, as long as you give appropriate credit to the original author(s) and the source, provide a link to the Creative Commons licence, and indicate if changes were made. The images or other third party material in this article are included in the article's Creative Commons licence, unless indicated otherwise in a credit line to the material. If material is not included in the article's Creative Commons licence and your intended use is not permitted by statutory regulation or exceeds the permitted use, you will need to obtain permission directly from the copyright holder. To view a copy of this licence, visit http://creativecomm ons.org/licenses/by/4.0/.

\section{References}

1. P.W. Wachulak, A. Bartnik, M. Skorupka, J. Kostecki, R. Jarocki, M. Szczurek, L. Wegrzynski, T. Fok, H. Fiedorowicz, Appl. Phys. B 111, 239-247 (2013)

2. M. Müller, T. Mey, J. Niemeyer, K. Mann, Opt. Express 22, 23489 (2014)

3. P. Wachulak, A. Torisi, A. Bartnik, D. Adjei et al., Appl. Phys. B 118(4), 573-578 (2015)

4. M. Kördel, A. Dehlinger, C. Seim, U. Vogt, E. Fogelqvist, J.A. Sellberg, H. Stiel, H.M. Hertz, Optica 7(6), 658-674 (2020). https://doi.org/10.1364/ OPTICA.393014

5. A. Bartnik, H. Fiedorowicz, R. Jarocki, J. Kostecki, M. Szczurek, P.W. Wachulak, Nucl. Instrum. Methods Phys. Res. A 647, 125-131 (2011)

6. C. Peth, F. Barkusky, K. Mann, J. Phys. D Appl. Phys. 41, 105202 (2008)

7. F.-C. Kühl, M. Müller, M. Schellhorn, K. Mann, S. Wieneke, K. Eusterhues, J. Vac. Sci. Technol. A 34, 041302 (2016)

8. I. Mantouvalou, K. Witte, W. Martyanov, A. Jonas, D. Grötzsch, C. Streeck, H. Löchel, I. Rudolph, A. Erko, H. Stiel, B. Kanngießer, Appl. Phys. Lett. (2016). https:// doi.org/10.1063/1.4951000

9. M. Nikl, P. Bruza, D. Panek, M. Vrbova et al., Appl. Phys. Lett. 102, 161907 (2013)

10. I.C.E. Turcu, C.M. Mann, S.W. Moon, R. Allott, N. Lisi, B.J. Maddison, S.E. Huq, N.S. Kim, Microelectron. Eng. 35(1-4), 541-544 (1997)

11. H.J. Levinson, Extreme Ultraviolet Lithography, in EUV Lithography, 2nd edn., ed. by V. Bakshi (SPIE Press, New York, 2018)

12. P. Choi, S.V. Zakharov, R. Aliaga-Rossel, V.S. Zakharov et al., J. Micro Nanolith. Mems. Moems. 11(2), 021107 (2012)

13. H. Fiedorowicz, A. Bartnik, R. Jarocki, J. Kostecki, J. Krzywiński, J. Mikołajczyk, R. Rakowski, A. Szczurek, M. Szczurek, J. Alloy. Compd. 401(1-2), 99-103 (2005)

14. H. Fiedorowicz, A. Bartnik, P. Parys, Z. Patron, Appl. Phys. Lett. 62, 2778 (1993) 
15. H. Fiedorowicz, A. Bartnik, M. Szczurek, H. Daido, N. Sakaya, V. Kmetik, Y. Kato, M. Suzuki, M. Matsumura, J. Tajima, T. Nakayama, T. Wilhein, Opt. Commun. 163, 103 (1999)

16. A. Bayer, F. Barkusky, S. Doring, P. Großmann, K. Mann, X-Ray Opt. Instrum. 2010, 687496 (2010)

17. G. O'Sullivan, B. Li, P. Dunne, P. Hayden, D. Kilbane,

R. Lokasani et al., Phys. Scr. 90, 054002 (2015)

18. R. Lokasani, C. Arai, Y. Kondo, H. Hara, T.-H. Dinh et al., Appl. Phys. Lett. 109, 194103 (2016)

19. M. Müller, F.-C. Kühl, P. Großmann, P. Vrba, K. Mann, Opt. Express 21, 12831 (2013)

20. P. Vrba, M. Vrbova, S.V. Zakharov, V.S. Zakharov, Phys. Plasmas 21, 073301 (2014)

21. V.S. Zakharov, S.V. Zakharov, J. Phys. D Appl. Phys. 53, 195202 (2020)

22. P. Vrba, M. Vrbova, M. Müller, K. Mann, D. Panek, T. Parkman, Phys. Plasmas 24, 123301 (2017)

23. H. Fiedorowicz, A. Bartnik, R. Jarocki, R. Rakowski, M. Szczurek, Appl. Phys. B 70, 305 (2000)

24. S.V. Zakharov, V.G. Novikov, P. Choi, Zstar Code for DPP and LPP Source Modeling in EUV Sources for Lithography, ed. by V. Bakshi (SPIE Optical Engineering Press, Bellingham), pp. 223-275

25. A.F. Nikiforov, V.G. Novikov, Quantum-Statistical Models of Hot Dense Matter Methods for Computation Opacity and Equation of State (Birkhaüser Verlag, Basel, 2005)

26. R. Benattar, S.V. Zakharov, A.F. Nikiforov, V.G. Novikov, V.A. Gasilov, A.Y. Krukovskii, V.S. Zakharov, Phys. Plasmas 6, 175 (1999)

27. V.G. Novikov, S.V. Zakharov, JQSRT 81, 339-354 (2003)

28. R. De Bruijn, K.N. Koshelev, S.V. Zakharov, V.G. Novikov, F. Bijkerk, Phys. Plasmas 12(04), 2701 (2005)

29. J.J. Duderstadt, G.A. Moses, Inertial Confinement Fusion (Wiley, New York, 1982)

30. M.V. Ammosov, N.B. Delone, V.P. Krainov, Sov. Phys. JETP 64, 1191-1194 (1986)
31. S.V. Zakharov, V.S. Zakharov, V.G. Novikov, M. Mond, P. Choi, Plasma Sources Sci. Technol. 17, 024017 (2008)

32. P. Wachulak, M. Duda, A. Bartnik, Ł Wegrzynski, T. Fok, H. Fiedorowicz, APL Photon. 4, 030807 (2019). https://doi.org/10.1063/1.5085810

33. V.S. Zakharov, J. Phys. D Appl. Phys. 50, 035202 (2016)

34. R.D. Cowan, The Theory of Atomic Structure and Spectra (University of California Press, Oakland, 1981)

35. M.F. Gu, Can. J. Phys. 86, 675-89 (2008)

36. S. Chandrasekhar, Hydrodynamic and Hydromagnetic Stability (Clarendon Press, Oxford, 1961) 